\title{
THE ELECTRON AND ION PLASMA EXPERIMENT FOR FAST
}

\author{
C. W. CARLSON, J. P. McFADDEN, P. TURIN and D. W. CURTIS \\ Space Sciences Laboratory, University of California, Berkeley. CA 94720, U.S.A.
}

A. MAGONCELLI

Lockheed Martin, Advanced Technology Center, Palo Alto, CA 94304, U.S.A

\begin{abstract}
The ion and electron plasma experiment on the Fast Auroral Snapshot satellite (FAST) is designed to measure pitch-angle distributions of suprathermal auroral electrons and ions with high sensitivity, wide dynamic range, good energy and angular resolution, and exceptional time resolution. These measurements support the primary scientific goal of the FAST mission to understand the physical processes responsible for auroral particle acceleration and heating, and associated waveparticle interactions. The instrument includes a complement of 8 pairs of 'Top Hat' electrostatic analyzer heads with microchannel plate (MCP) electron multipliers and discrete anodes to provide angle resolved measurements. The analyzers are packaged in four instrument stacks, each containing four analyzers. These four stacks are equally spaced around the spacecraft spin plane. Analyzers mounted on opposite sides of the spacecraft operate in pairs such that their individual $180^{\circ}$ fields of view combine to give an unobstructed $360^{\circ}$ field of view in the spin plane. The earth's magnetic field is within a few degrees of the spin plane during most auroral crossings, so the time resolution for pitch-angle distribution measurements is independent of the spacecraft spin period. Two analyzer pairs serve as electron and ion spectrometers that obtain distributions of 48 energies at 32 angles every $78 \mathrm{~ms}$. Their standard energy ranges are $4 \mathrm{eV}$ to $32 \mathrm{keV}$ for electrons and $3 \mathrm{eV}$ to $24 \mathrm{keV}$ for ions. These sensors also have deflection plates that can track the magnetic field direction within $10^{\circ}$ of the spin plane to resolve narrow, magnetic field-aligned beams of electrons and ions. The remaining six analyzer pairs collectively function as an electron spectrograph, resolving distributions with 16 contiguous pitch-angle bins and a selectable trade-off of energy and time resolution. Two examples of possible operating modes are a maximum time resolution mode with 16 angles and 6 energies every $1.63 \mathrm{~ms}$, or a maximum energy resolution mode with 16 angles and 48 energies every $13 \mathrm{~ms}$. The instrument electronics include mcp pulse amplifiers and counters, high voltage supplies, command/data interface circuits, and diagnostic test circuits. All data formatting, commanding, timing and operational control of the plasma analyzer instrument are managed by a central instrument data processing unit (IDPU), which controls all of the FAST science instruments. The IDPU creates slower data modes by averaging the high rate measurements collected on the spacecraft. A flexible combination of burst mode data and slower 'survey' data are defined by IDPU software tables that can be revised by command uploads. Initial flight results demonstrate successful achievement of all measurement objectives.
\end{abstract}

\section{Introduction}

The primary scientific objectives of the FAST mission are to understand the microphysics of the aurora that lead to acceleration of electron and ion beams, heating of ion conics, and generation of a multitude of wave/particle interactions (Temerin 
et al., 1990; Carlson et al., 1998a). FAST instruments were specifically designed to obtain accurate, high resolution particle and field measurements on extremely short temporal and spatial scales. They are motivated by earlier instruments flown on sounding rockets that first resolved intense field-aligned electron beams and the rapidly modulated electron oscillations that cause flickering aurora ( $\mathrm{McFad}-$ den et al., 1986, 1987). Recent measurements by the TESP instrument on Freja (Boehm et al., 1994) also demonstrate the value of fast time resolution electron measurements at the lower edge of the acceleration region. FAST uses an integrated package of particle and fields instrumentation to make measurements at higher altitudes, within the heart of the auroral acceleration region, up to an apogee altitude of $4200 \mathrm{~km}$. These measurements are revealing the origin of phenomena that were discovered on earlier spacecraft but not fully resolved or understood, such as suprathermal bursts and counterstreaming electron beams (Sharp et al., 1980), ion conics (see, e.g., a review by Klumpar, 1986), auroral kilometric radiation (Gurnett et al., 1974), and electrostatic shocks (Mozer et al., 1977). Understanding waveparticle interactions quantitatively requires not only high time resolution, but also sufficient accuracy to compute wave growth and damping.

The FAST satellite is designed to acquire 'snapshots' or bursts of high speed data that cannot be transmitted continuously, as well as continuous 'survey data' covering the entire auroral pass. The instruments are designed to function as a single coordinated experiment to acquire complementary particle and fields measurements. A variety of data modes combine selected measurements from all of the instruments on several time scales. A typical auroral crossing includes 'slow survey' data where particle precipitation is low, 'fast survey' data when significant electron energy flux is detected, and 'burst' data with full time resolution when various criteria of high activity are found. The decision for switching modes is triggered by automatic on-board evaluation of the measurements. Additional discussion of the FAST data processing system and the fields data triggers are described by Harvey et al. (this issue) and Ergun et al. (this issue).

The FAST particle instruments include the electron and ion plasma instrument described here, which emphasizes rapid measurements of electron and ion pitchangle distributions, and the TEAMS instrument (Klumpar et al. (this issue)), which provides complementary ion composition measurements of the full three dimensional distribution function of dominant auroral ions with a time resolution of 2.5 seconds. The following instrument description is presented in two parts. The overview section provides a general discussion of the instrument operation that is useful for interpreting the data products. The more technical information is presented in the later sections. 


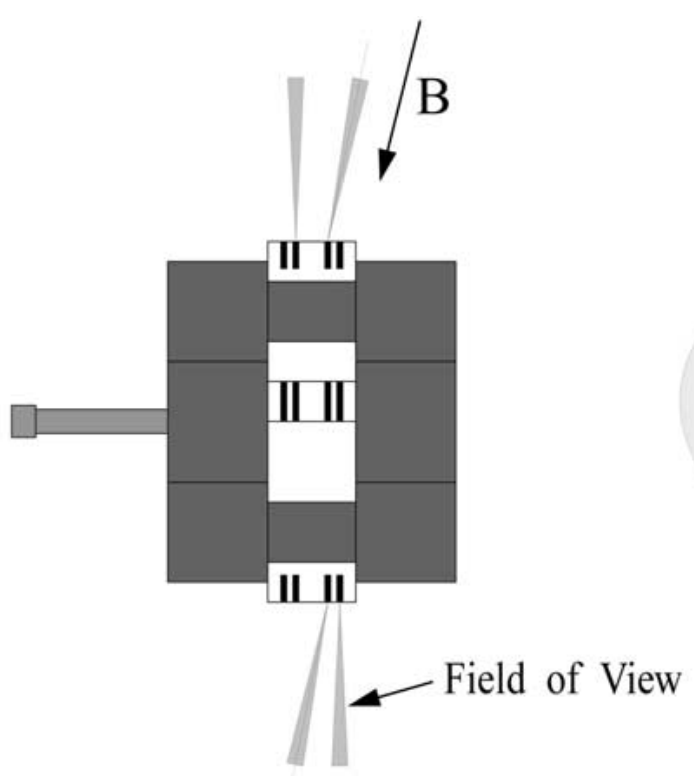

SIDE VIEW

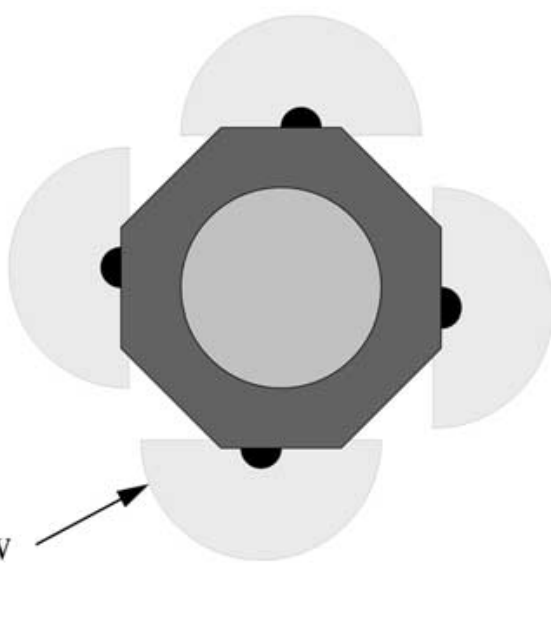

END VIEW

Figure 1. Two views of the FAST satellite illustrating the location of the four ESA stacks and their fields of view. Each stack contains four ESA sensors that are paired with a complementary stack on the opposite side of the deck to provide $360^{\circ}$ radial fields of view. Deflectors at the entrance to the EESA and IESA pairs allow their fields of view to be aligned with the magnetic field as shown in the side view. The side view only illustrates four sensor fields of view for simplicity.

\section{Instrumentation}

\subsection{INSTRUMENT OVERVIEW}

The design challenge for the fast plasma instrument is to obtain continuous high resolution measurements of the electron and ion pitch-angle distributions with sub-second time resolution. Our design takes advantage of a property of the high inclination orbit to obtain continuous viewing of all pitch-angles. The FAST orbit has an $83^{\circ}$ inclination, so the earth's dipole magnetic field forms a small angle to the orbital plane. The magnetic orientation of the FAST spacecraft axis is optimized by tilting the spacecraft spin axis out of the orbit normal by about $3^{\circ}$ toward alignment with the earth's axis. With this orientation, the earth's magnetic field remains within $10^{\circ}$ of the FAST spin plane throughout most high latitude auroral passes, so continuous pitch angle measurements can be accomplished by aligning detector fields of view with the spacecraft spin plane.

Figure 1 illustrates examples of representative fields of view of the FAST plasma instrument, which includes sixteen Top Hat electrostatic analyzers arranged in four 'stacks' of four analyzers each, mounted symmetrically around the central instrument deck. Each analyzer has a fan-shaped $180^{\circ}$ field of view in the spacecraft spin 
TABLE I

Fast particle detectors

\begin{tabular}{lccccccc}
\hline Measurement & $\begin{array}{c}\text { Energy } \\
\text { range }\end{array}$ & $\Delta E / E$ & $\begin{array}{l}\text { Sampling } \\
\text { resolution }\end{array}$ & $\begin{array}{l}\text { Field } \\
\text { of view }\end{array}$ & $\begin{array}{l}\text { Angular } \\
\text { resolution }\end{array}$ & Sample array & $\begin{array}{l}\text { Geometric } \\
\text { factor } \\
\left(\mathrm{cm}^{2} \mathrm{sr} E / E\right)\end{array}$ \\
\hline $\begin{array}{l}\text { Ion } \\
\text { spectrometer }\end{array}$ & $5 \mathrm{eV}-24 \mathrm{keV}$ & 0.20 & $78 \mathrm{~ms}$ & $360^{\circ} \times 12^{\circ *}$ & $11.2^{\circ} \times 12^{\circ}$ & $48 \mathrm{E} \times 32 \alpha$ & 0.009 \\
$\begin{array}{l}\text { Electron } \\
\text { spectrometer }\end{array}$ & $6 \mathrm{eV}-30 \mathrm{keV}$ & 0.15 & $78 \mathrm{~ms}$ & $360^{\circ} \times 10^{\circ *}$ & $11.2^{\circ} \times 10^{\circ}$ & $48 \mathrm{E} \times 32 \alpha$ & 0.005 \\
$\begin{array}{l}\text { Electron } \\
\text { Spectrograph }\end{array}$ & $6 \mathrm{eV}-30 \mathrm{keV}$ & 0.15 & $1.6 \mathrm{~ms}$ & $360^{\circ} \times 10^{\circ}$ & $22.5^{\circ} \times 10^{\circ}$ & $6 \mathrm{E} \times 16 \alpha^{* *}$ & $6 \times 0.01$ \\
\hline
\end{tabular}

*Field of view can deflected \pm 10 deg from the center angle.

**A larger energy sample array can be achieved with poorer time resolution.

plane, and analyzers located on opposite sides of the spacecraft operate as pairs to obtain a full $360^{\circ}$ view. The main solar panels are body-mounted on octagonal structures located above and below the instrument deck. Additional small solar panels fill areas between instruments on the instrument deck.

For simplicity, the magnetometer and electric field booms are omitted from this figure. These booms mount to the instrument deck and are located to cause minimum interference with the particle detectors. The electric field booms in the spin plane are thin wires that are located between the entrance apertures of the analyzers. The exterior conductor of the boom is at spacecraft potential and the wire obscures a negligible portion of the field of view. Likewise, the magnetometer booms are located to obscure a minimum solid-angle within the plasma instrument field of view. Further description and diagrams of the booms and their configuration on the spacecraft are presented in companion papers (Pfaff et al. (this issue); Pankow et al. (this issue)).

The FAST plasma analyzers use a common design that is configured for three specific applications to optimize either high energy and angle resolution or high time resolution. The basic analyzer parameters for these three options are summarized in Table I. Two of the analyzer pairs operate as swept energy spectrometers to measure electron (EESA) and ion (IESA) distributions, emphasizing energy and pitch-angle resolution. Each of the detector stacks includes one of these spectrometer analyzers, which have 16 azimuth angle sectors and typically sample 48 energy bins spanning energies from $4 \mathrm{eV}$ to $32 \mathrm{keV}$ for electrons and $3 \mathrm{eV}$ to $24 \mathrm{keV}$ for ions. Spacing of energy samples is designed for approximately constant $\Delta E / E$ spectral resolution. The nominal energy sweep rate of 12.8 sweeps $\mathrm{s}^{-1}$ is phaselocked to produce exactly 64 sweeps during the 5-s spin period of the spacecraft. The standard accumulation time at each energy step is approximately $1.63 \mathrm{~ms}$. Other selectable sweep options include a double-speed mode with 24 energy steps, and a half-speed, high resolution sweep with 96 steps. Each sweep cycle requires 
one retrace step so the number of resolved energy levels is one fewer than the number of steps in the sweep.

The spectrometers include deflection plates that independently steer the field of view of each analyzer up to $10^{\circ}$ above or below the spin plane to track the magnetic field direction, creating continuous coverage of all pitch angles. As Figure 1 illustrates, the deflections of the upward and downward viewing analyzers are in opposite directions to allow simultaneous viewing in both directions along the magnetic field. This capability is essential for investigating magnetic field-aligned electron and ion beams, which may travel in either direction along the magnetic field. The central Instrument Data Processor Unit (IDPU), (see Harvey et al., this issue), uses magnetometer data to automatically compute deflection angles and command the analyzer deflector plate voltages.

The geometric factors for the EESA and IESA sensors listed in Table I are consistent with the energy sweep times and counting rate capabilities of the detector electronics. The electron differential energy fluxes of auroral electrons typically range between $10^{7}$ and $10^{10} \mathrm{eV} / \mathrm{cm}^{2}$-s-sr-eV. Each anode in the EESA has a geometric factor of $1.6 \times 10^{-4} \mathrm{~cm}^{2}-\mathrm{sr}-\mathrm{eV} / \mathrm{eV}$, so typical count-rates range between $10^{3}$ and $10^{6}$ per second per anode. The pulse amplifiers have a deadtime of $140 \mathrm{~ns}$, so these rates are easily accommodated, and the MCP gain and strip current are designed for $5 \mathrm{MHz}$ rates. The standard accumulation time of $1.6 \mathrm{~ms}$ thus obtains samples ranging between 1 and $10^{3}$ counts. The accumulator circuits have 14 bits, for a capacity of $1.6 \times 10^{4}$ counts. Lower fluxes are easily identified by averaging multiple sweeps. The IESA sensor has a somewhat larger geometric factor to match the lower expected ion fluxes.

The EESA/IESA sensors provide a good combination of energy, angle, and time resolution that form the baseline plasma particle measurements. They are typically operated in a standard mode that covers the entire energy range at full time resolution. The total volume of data are compressed for summary data by averaging these measurements in the IDPU.

The remaining six pairs of analyzers are electron sensors that collectively function as a spectrograph (SESA), using a variety of programmable energy scan cycles to accomplish the highest time resolution measurements. Each of the SESA analyzers has 16 pitch angle sectors and can operate over the same 48 step energy range as the EESA spectrometer and uses the standard $1.63 \mathrm{~ms}$ accumulation cycle. This parallel detection design uses a total of 96 separate angle sector anodes with individual amplifier/accumulator strings. The large geometric factor $\left(0.06 \mathrm{~cm}^{2}\right.$-sr$\mathrm{eV} / \mathrm{eV}$ ) of the SESA instrument is an order of magnitude larger than the EESA, allowing it to make electron distribution measurements with unprecedented time resolution and good counting statistics. The geometric factor for each SESA anode is four times larger than for the EESA. Thus the count-rates for intense beams with fluxes of $10^{10} \mathrm{eV} / \mathrm{cm}^{2}$-s-sr-eV will be $4 \times 10^{6}$ per anode, which is consistent with the design limit of the amplifiers, accumulators, and MCP saturation level. 
TABLE II

Weight and power

\begin{tabular}{lcc}
\hline & Per Stack & Total \\
\hline Mass & $3.85 \mathrm{~kg}$ & $15.4 \mathrm{~kg}$ \\
Power: & & \\
LV & $1.8 \mathrm{~W}$ & $7.2 \mathrm{~W}$ \\
HV & $1.2 \mathrm{~W}$ & $4.8 \mathrm{~W}$ \\
Total & $3.0 \mathrm{~W}$ & $12 \mathrm{~W}$ \\
\hline
\end{tabular}

A variety of hardware programmed operating modes are used with the SESA. They can operate in a full energy spectrometer mode, similar to the EESA, with each analyzer sweeping over the complete energy range, but with a phase shift between each of the six pairs. This mode generates full energy spectra every $13 \mathrm{~ms}$, six times faster than EESA, but with half the angle resolution. Faster modes are accomplished by dividing the energy range among the six analyzer pairs and reducing the number of energy steps for each analyzer. For example, a 24 step energy spectra (4 steps per analyzer) can be collected every $6.5 \mathrm{~ms}$, a 12 step spectrum requires $3.3 \mathrm{~ms}$, or each SESA pair can remain at fixed energies, yielding a six point spectrum every $1.63 \mathrm{~ms}$. The energy range for these cycle modes can be selected to span the entire energy range, or can be concentrated in narrower ranges. One application using a narrow energy range is the 'peak tracking mode', which uses the EESA data to identify a peak in the energy distribution and then slaves the SESA to make rapid measurements in a limited energy range surrounding the peak. Table I summarizes the possible combinations of energy and time resolution for the EESA and SESA.

Operating modes for the ESA sensors are programmed in hardware. The raw measurements produced by the ESA detectors represent the highest speed burst data that are transmitted to the ground. Lower rate survey data products are generated in the IDPU by time-averaging particle distributions according to selected software modes. The ESA hardware modes are typically fixed throughout a single auroral zone pass, whereas the IDPU may have several levels of data compression that are adaptively selected according to onboard data evaluation algorithms. For example, the fast survey, slow survey, and burst modes are determined by evaluations of measured data performed by the IDPU. The IDPU always generates the plasma survey data with at least two distributions per spin that are available at one spin resolution in CDF (Common Data Format) files available from the NSSDC (National Space Science Data Center) or as summary data plots from the FAST web site. 


\subsection{DESIGN CONSIDERATIONS}

The FAST mission imposed severe constraints on the spacecraft and instrument designs. The science requirements demanded very capable instruments generating exceptionally high data rates that would fit within the cost and launch capability of a Small Explorer mission. The instrument designs had to minimize weight, power, and volume, and tolerate a high radiation environment to attain the desired orbit. Rather than a 'box on deck' design, FAST instruments followed a more integrated design where instrument boxes and solar panels form a complete radiation and thermal close-out of the instrument deck. With this design, interior components receive a minimum radiation shielding equivalent of $4 \mathrm{~mm}$ of aluminum. Since the plasma instrument must be exposed to the outside, its design ensured that all electronic components were located behind passive structure or explicit shielding. The electric field boom mechanisms are also integrated with the analyzer stacks to increase shielding, conserve weight, and provide a single interface to the spacecraft. The weight and power requirements for individual stacks and for the total instrument are listed in Table II.

Because the plasma instrument uses a large number of analyzers, their design criteria emphasized ease of fabrication and testing. The detectors are composed of modular components that can be assembled and tested separately and are easily replaced if necessary. The design also provides contamination control features including sealed apertures and a dry nitrogen purge system.

\subsection{Detector Design}

The main elements of the plasma detector design are the particle optics design, the mechanical design of the electrostatic analyzers, the electrical and mechanical design of the MCP anode boards, and the contamination control system.

\subsubsection{Analyzer Optics}

The electrostatic analyzers used on FAST are spherical section top-hat analyzers (Carlson et al., 1983; Carlson and McFadden, 1998) with a uniform $180^{\circ}$ field of view. As shown in Figure 2, each analyzer consists of an inner hemisphere mounted on an insulating plastic ring, and a concentric outer hemisphere with a circular entrance hole at the top. A third concentric top-hat electrode is suspended above the entrance hole. An attracting voltage is applied to the inner hemisphere which allows charged particles with the corresponding energy per charge to pass through the analyzer and be detected at the microchannel plate (MCP) electron multiplier. An entrance aperture in the cylindrical analyzer housing collimates the particle beam and also restricts entry of solar UV radiation into the analyzer. Collimation apertures are also located near the entrance to the top-hat region, and the top-hat entrance itself defines the final collimation. Conical deflection plates are included behind the entrance collimator of the EESA/IESA analyzers to steer the detector field of view by $\pm 10^{\circ}$. Typical particle trajectories in each analyzer are shown. 


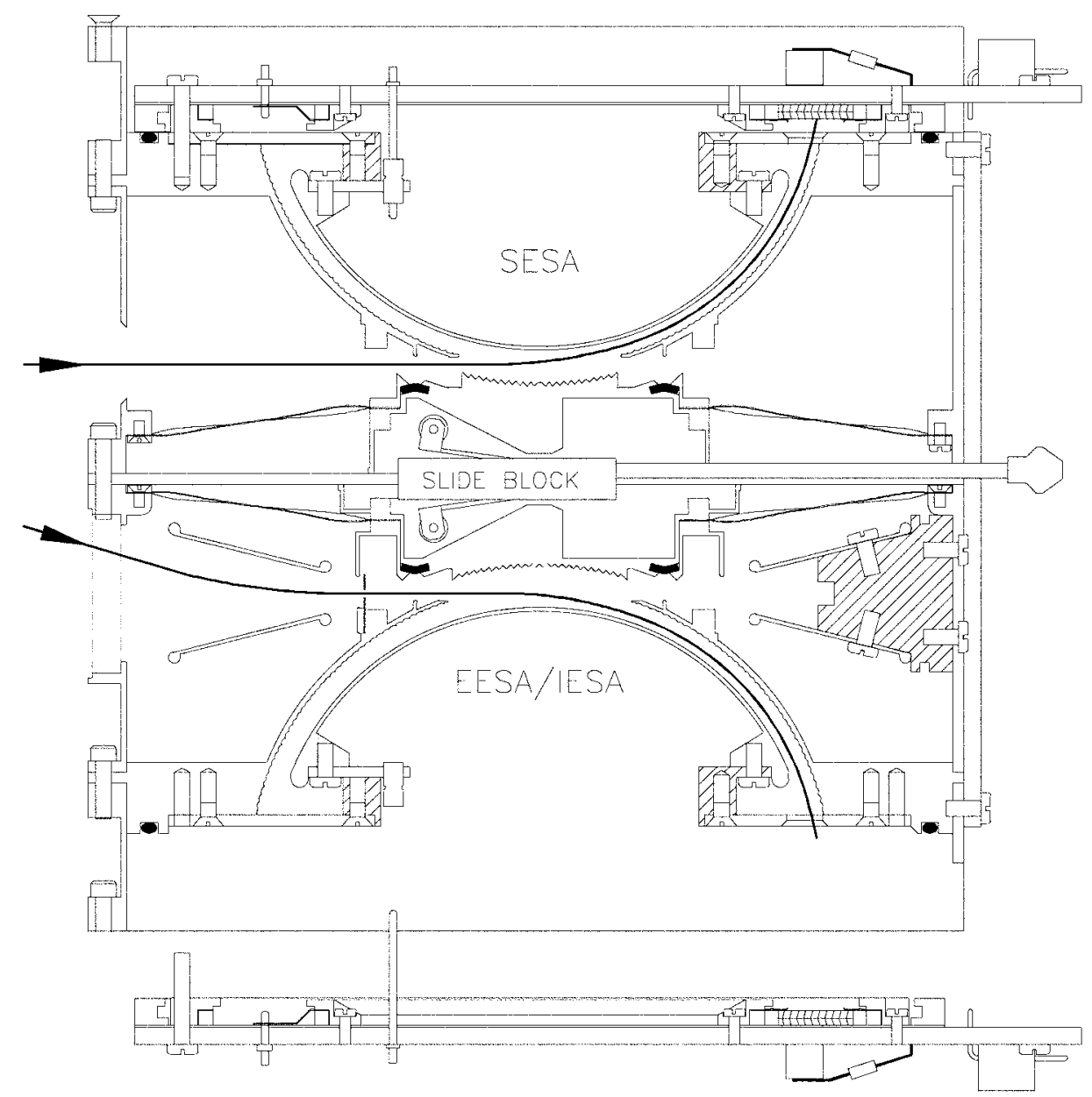

Figure 2. A cut-away drawing of an ESA pair module, illustrating the hemisphere mounting configuration, the top-hat closing mechanism, and the MCP anode mounting. Particle trajectories show the deflection through the analyzer, and also the operation of the entrance deflection plates that allow these sensors to continuously measure magnetic field-aligned fluxes.

Because negatively charged surfaces might generate accelerated photoelectrons, the deflection systems use only positive voltages to either attract electrons or repel ions.

The particle optics response for the FAST analyzers was thoroughly characterized by computer simulations (McFadden and Carlson, 1998) and with vacuum chamber calibrations. The simulated response, plotted in Figure 3(a), illustrates the typical angle/energy skew expected for a $90^{\circ}$ deflection analyzer. Simulations are compared with measured data for integrated energy and angle responses in Figures 3(b) and 3(c). The energy response in Figure 3(b) was measured with an electron beam energy of $10 \mathrm{keV}$. The overlapping traces at the bottom of the 


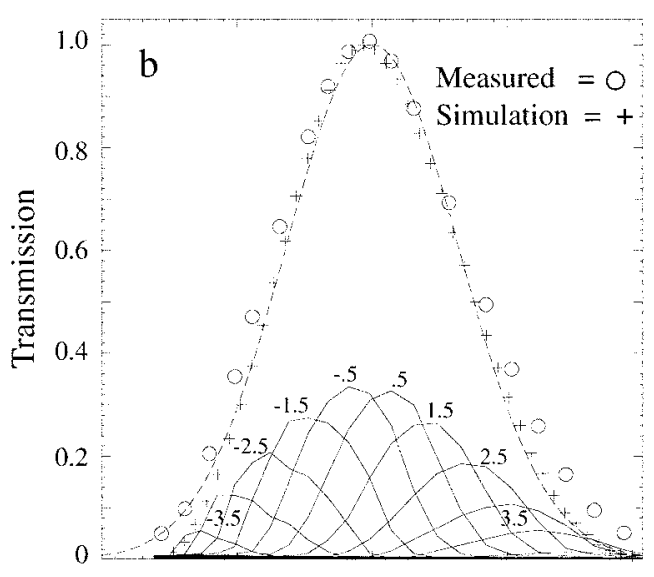

FAST

Electron ESA
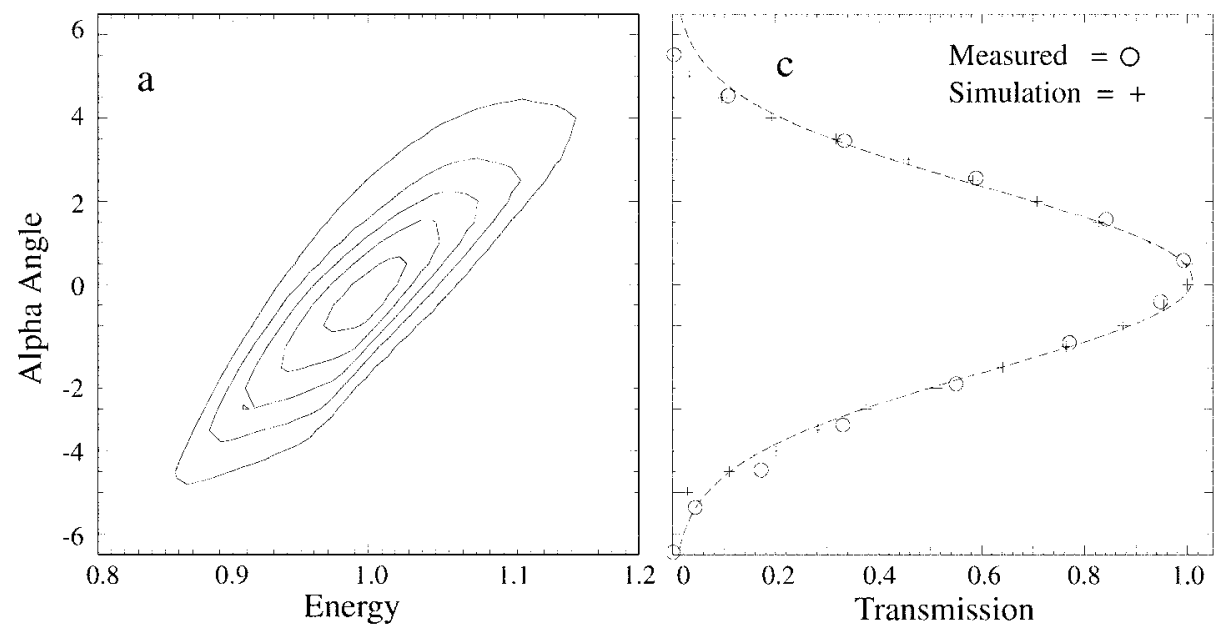

Figure 3. (a) The FAST EESA energy-angle response. The energy-angle contour plot is the simulated analyzer response. (b) The simulated and measured energy response averaged over angles. (c) The simulated and measured angle response averaged over incident energy.

plot are energy scans at each $1^{\circ}$ increment of elevation angle $\alpha$. The measured response integrated over $\alpha$ is plotted as open circles. The computer simulation data are shown with + symbols, which have also been fitted with a Gaussian function denoted with a dashed line. The measured response shows good agreement with the simulation, but is slightly wider on the high energy wing. Measurements using ion beams did not show this widening, so we attribute the wider electron response to small angle forward scattering of electrons hitting the outer hemisphere. The analyzer response parameters summarized in Table I are derived from the analyzer simulations. 


\subsubsection{Analyzer Mechanical Design}

The most critical mechanical requirement for the analyzer design is the stable and accurate alignment of the three hemispherical electrode surfaces. Requirements from other considerations are ease of fabrication and a simple interface to other components making up the complete instrument. The inner and outer hemispheres and the top-hats are machined from aluminum. Figure 2 illustrates most of the mechanical components of the analyzers. The electrode surfaces of the top-hats and the outer hemispheres have a scalloped finish that reduces scattering of particles and UV photons that can reach the exit aperture and produce background events. The hemispheres and top-hat components were gold plated to ensure good surface electrical properties, and a gold-black coating was vacuum deposited on their electrode surfaces to further reduce particle and UV scattering. Other analyzer surfaces are painted with conductive paint to ensure good surface conductivity.

The mechanical alignment of the analyzer components is maintained by using a small number of simple parts in the optical system. The outer hemisphere is machined from a single piece of aluminum that also forms the main mechanical structure of the analyzer assembly. A flat circular exit aperture plate is fastened to the hemisphere base and extends under the inner hemisphere. The aperture plate has an annular exit aperture that extends approximately $180^{\circ}$. An etched $\mathrm{BeCu}$ grid covers the exit aperture to terminate the analyzer electric field at the aperture plane. An insulating ring made of PEEK connects the inner hemisphere to the aperture plate. PEEK is a very stable, high temperature plastic with low outgassing properties. The plastic ring has a series of vertical slots machined about half way through the vertical height of the ring to accommodate differential thermal expansion between it and the aluminum parts without affecting the axial alignment. The fabrication tolerances for the hemispheres, aperture plate, and plastic ring are tightly controlled, and the combination of flat and cylindrical alignment surfaces provide stable positioning in both the axial and radial directions.

The alignment of the top-hat is also determined by a simple mechanical path. The outer hemisphere base is fastened directly to the external cylindrical tube that contains the entrance aperture collimator. A single flat aluminum plate fastens to the top of the tube and forms the common interface between the two halves of the analyzer module. A spoked spring suspends and centers the top-hat assembly within the cylinder housing. This suspension spring pulls the top-hat upward against the center plate, which determines the vertical position of the top-hat. The suspension spring is part of the aperture closing mechanism that is described later. The lower analyzer in Figure 2 illustrates the deflection plate mounting for the I/EESA. Two blocks of PEEK plastic provide a cantilever mounting of the analyzer plate assembly to the cylinder body. A double mesh grid covers the entrance aperture at the front of the analyzer housing to define the electrical potential boundary conditions for the deflector optics and also prevent leakage of the potential into the external plasma. 


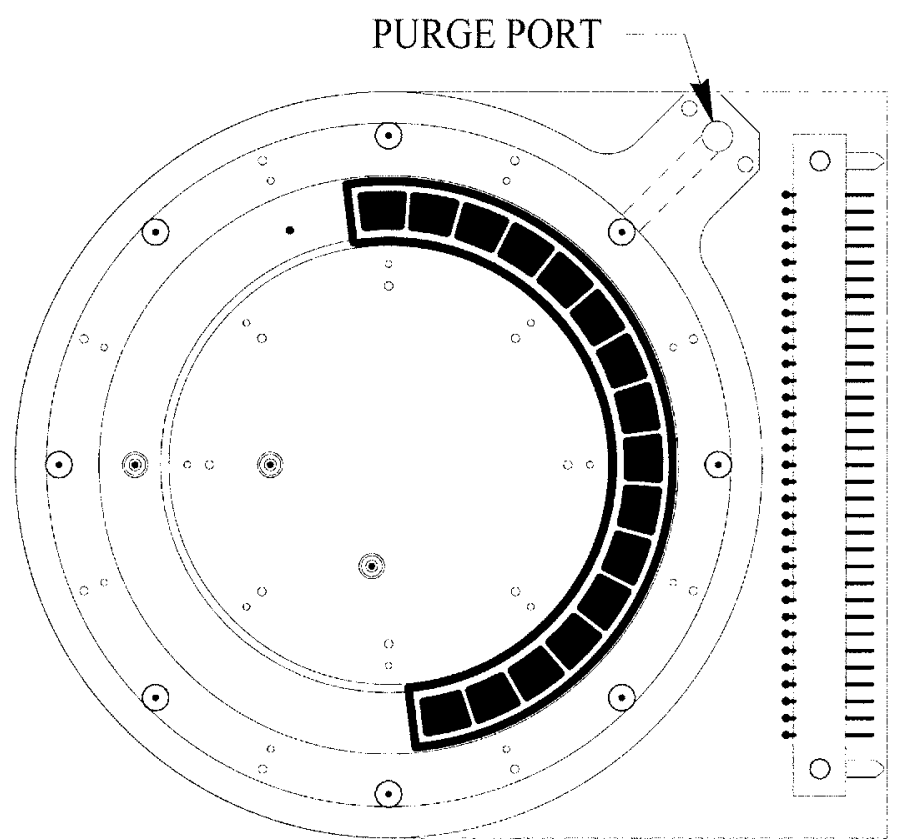

Figure 4. A mechanical drawing of the EESA/IESA anode board showing the configuration of the charge collection pads, the MCP electrical contact, and the purge port used for the nitrogen purge system.

The final components comprising the structure of the analyzer assembly are the top and bottom interface rings. These components have a ' $D$ ' shape when viewed along the analyzer axis, and they serve to join the cylindrical analyzer assemblies to the rectangular housing for the detector electronics. They are also the housing for the MCP anode board assembly, which is shown in two configurations in Figure 2. The lower anode board is detached from the analyzer to show how it appears as a separate subassembly. It is normally installed in the analyzer with its MCP aligned with the exit aperture. The anode board also has a pin that connects the sweep high voltage to a socket on the inner hemisphere. The top analyzer is shown with its anode board installed. A Viton 0-ring makes a seal between the anode board and the analyzer base for contamination control.

\subsubsection{MCP Anode Board Assembly}

The anode board assembly includes the MCP mounting hardware, the signal anode pads, and the electrical circuitry needed to route the signals and high voltages to connectors. It is designed and fabricated using standard multilayer printed circuit technology. The substrate is a low outgassing polyimide board material that can be baked out at high temperatures.

The anode patterns and the general mechanical layout of the 16 anode EESA board is shown in Figure 4. The anode pads with their signal routing traces are 


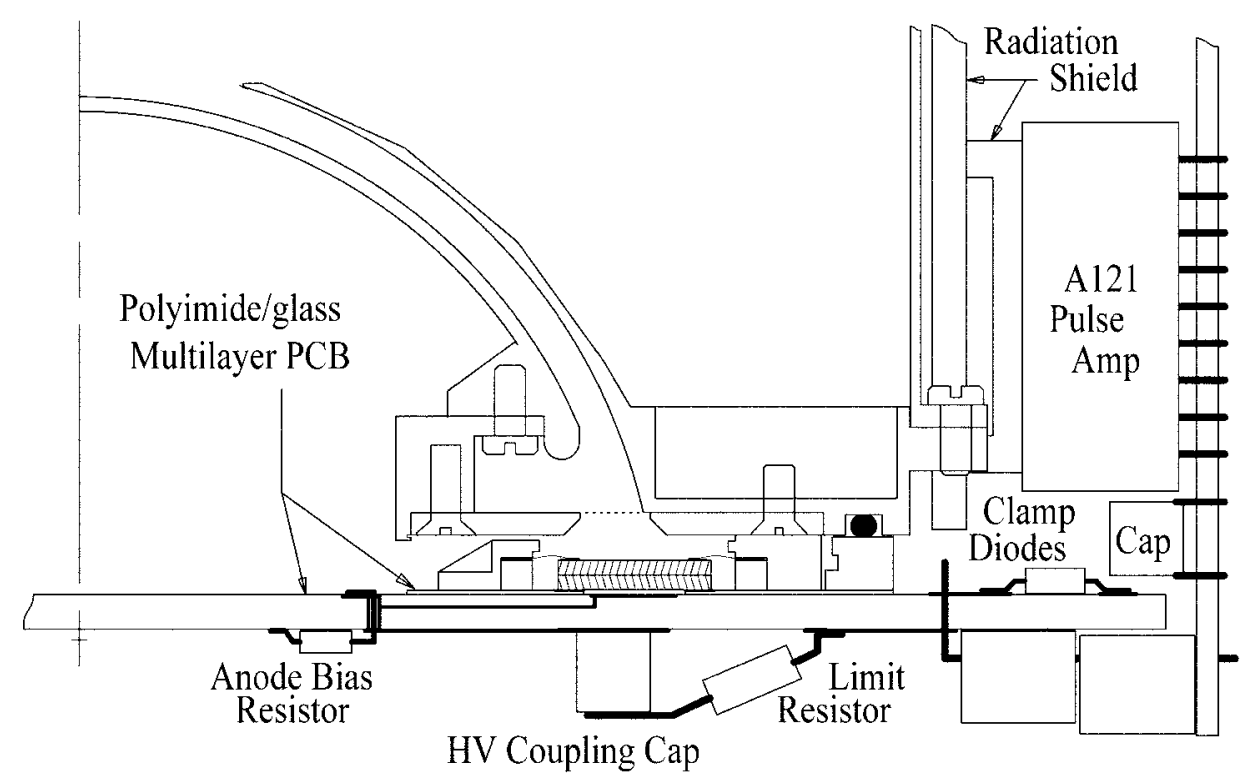

Figure 5. A cut-away view of the ESA analyzer showing the mechanical mounting of the MCPs and the electrical signal path from the anodes to the preamplifiers.

located on an internal layer of the pc card. A semi-circular window through the top layer of the pcb exposes the anode pads to the MCP, which mounts on the metal trace surrounding the anode window. The thickness of the top layer of the pcb lamination is $0.4 \mathrm{~mm}$ thick, which establishes the gap between the MCP and anode surface. The cut-away view in Figure 5 shows mechanical and electrical details of the anode board and its interfaces to the electrostatic analyzer and preamp electronics board. The anode pads are located at the bottom of the small gap below the MCPs. A buried trace connects each anode to a bias resistor that provides the dc path for anode current. The signal then passes through a HV coupling capacitor and a surge limit resistor to the signal connector that mates with the pulse amplifier board. The coupling capacitors are omitted for the ion instruments since the anodes are at low DC potential. Two diodes provide a bipolar voltage clamp on each signal trace to suppress any voltage spikes caused by sparking or corona. This figure also illustrates the close proximity of the pulse amplifiers to the anodes, their radiation 'spot shielding', and the mounting of the amplifier boards to the analyzer modules. High voltage connectors on each anode board plug directly into high voltage supplies. They are not included in Figure 5 but are shown in the exploded assembly drawing (Figure 6).

The two MCPs are mounted as a chevron pair with no inter-plate gap. The plates have 25 micron pores with a length/diameter ratio of 40:1. A rather high strip current of $5 \mu \mathrm{A} \mathrm{cm}{ }^{-2}$ is chosen for high count-rate capability. Imaging applications often include a gap between the plates to increase the MCP gain, but 


\section{FAST ESA Exploded View}

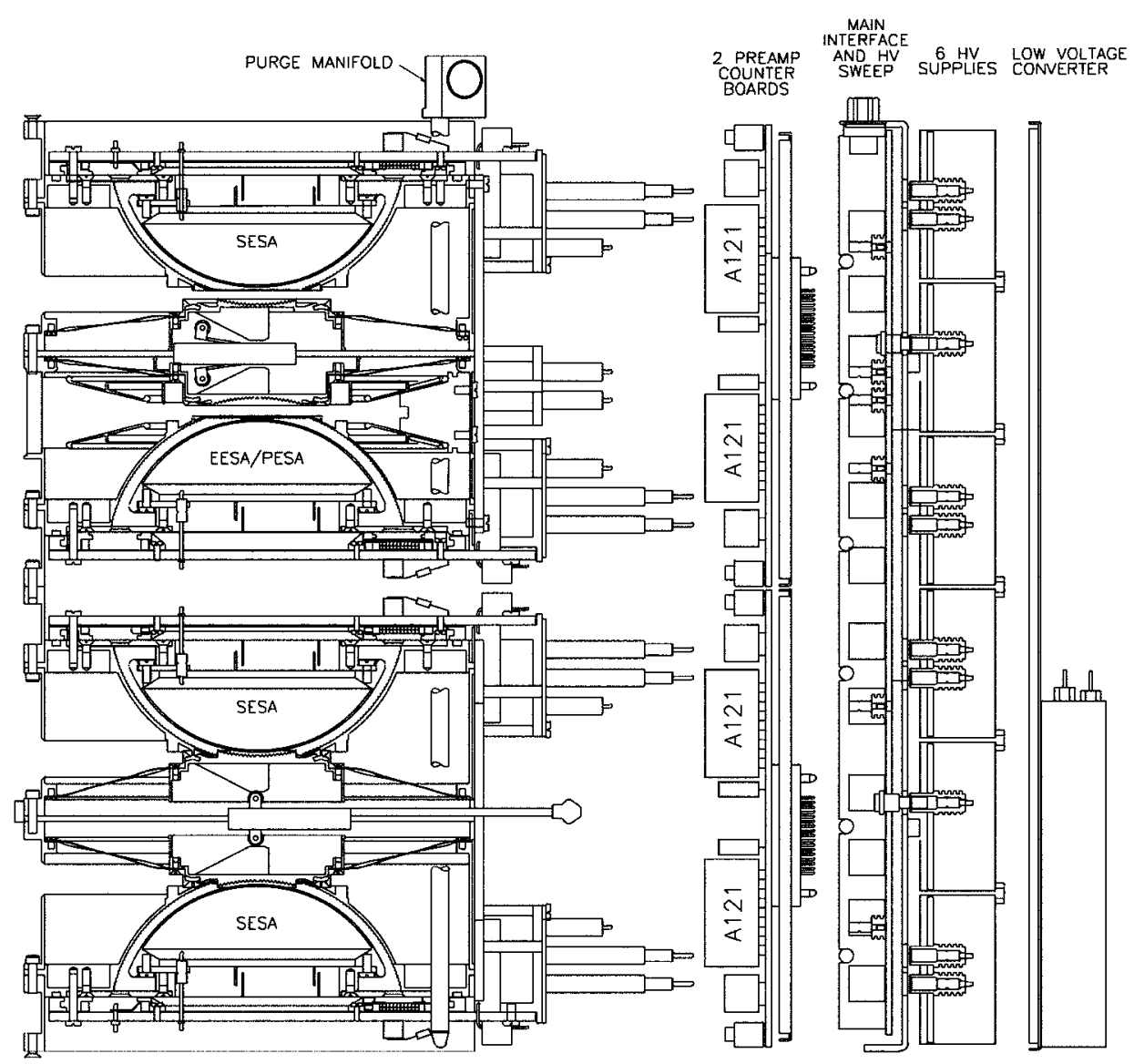

Figure 6. An exploded view of the FAST ESA stack assembly showing the stack of four analyzers, preamplifier boards, the interface and HV sweep control board, and the high and low voltage power converters. The modular design allows easy assembly for servicing and refurbishment.

that configuration also degrades the pulse-height distribution and increases gain variations between different positions on the plate. Image resolution for the FAST system is determined by individual anodes, and the design goal is maximum count rate capability. For these conditions excess MCP gain is a disadvantage, because it reduces the maximum count rate before onset of MCP saturation. The tightly clamped MCP configuration used for FAST optimizes uniform electron gain and narrow pulse height distributions with a modal gain of $1-2 \times 10^{6}$ electrons. The $\mathrm{MCP}$ and preamps are designed to handle maximum count rates of $5 \mathrm{Mhz}$ per anode segment without significant drop in detection efficiency.

The details of the MCP mechanical mounting are shown in Figure 5. Circular plastic rings, screwed to the anode board on each side of the MCP annulus, hold 
rings of Be-Cu spring fingers that clamp the MCP to the board. This clamping holds the MCPs in tight contact and also provides a good thermal path from the plates to the anode board. Because the channelplates have a negative thermal coefficient of resistance, high current applications such as FAST must be designed carefully to avoid thermal runaway. The outer MCP mounting ring is also the surface that contacts the analyzer aperture plate when the anode board is installed. The screws attaching the anode board to the analyzer base pass through this mounting ring. A concentric polyimide stiffener ring is bonded to the anode board just outside the outer mounting ring. An o-ring seals the joint between the stiffener ring and the analyzer base, so the only opening to the volume surrounding the MCP is the top-hat aperture. There is also access to introduce purge gas through a radial hole drilled in the edge of the stiffener ring (see Figure 4). Details of the purge system are presented in the next section.

\subsubsection{Contamination Control}

Microchannel plates are susceptible to contamination by particulates, and are also damaged by absorption of water vapor. The best storage environment for the MCP is either in a vacuum or a purged 'dry box'. The FAST analyzer modules provide similar protection for the MCP during storage and launch by including a nitrogen purge system for the sensors and using the top-hat assembly to seal the interior volume of the analyzer.

Figure 2 shows the main elements of the top-hat closer mechanism. Suspension springs center the two top-hats and pull them against the central mounting plate to hold them open during normal operation. The closer mechanism has spring-loaded rollers mounted on a slide-block that push against the inclined ramps machined into the interior of the top-hats. Horizontal motion of the slide-block thus pushes the two top-hats outward until the annular gaskets in the top-hats make contact and seal against thin circular protrusions on the outer hemisphere surface. The spring force on the rollers allows the top-hats to vent gas at a moderate over-pressure. The curved top-hat electrode protrudes into the hole in the outer hemisphere when the the closer is engaged. The slide-block is attached to a spring-loaded actuator rod that is released by a melt wire to open the top-hat seal on orbit.

The MCPs are considered the most vulnerable component for contamination, so the purge system is designed to deliver purge gas directly to the interior of the analyzer via the anode board. The spacecraft includes plumbing to distribute pressurized $\mathrm{N}_{2}$ gas to the particle instruments. The gas is supplied to each analyzer through a metal 'skewer' tube that passes though a small o-ring gland mounted on the corner of each anode board (see Figure 4). The skewer tube has small manifold holes aligned with each of these glands. The purge channel in each anode board contains a sintered filter and a small metering orifice that regulates the gas flow rate to each analyzer. 


\subsubsection{Stack Assembly}

Each of the four instrument stack assemblies contains three SESAs and either an ion or electron spectrometer (IESA or EESA). The stacks are composed of two major sub-assemblies that can be assembled and tested individually, and the design of each sub-assembly is also modular. The exploded view of the instrument stack (Figure 6) illustrates the modules that make up the instrument stack. The analyzers are grouped in two dual analyzer modules with anode boards. This figure shows the anode board with their high voltage plugs that were missing from Figure 5. A counter/preamplifier board is mechanically mounted to each analyzer module and has connectors that mate to the two anode boards. A thin aluminum sheet is mounted to the top surface of the preamp board to shield it from electromagnetic noise on the main interface board. The sensor subassembly can be tested as a unit using external high voltage supplies and a simple digital test interface. Initial vacuum chamber testing of the analyzer assembly was done at this level.

The second major subassembly includes the high voltage and interface functions and contains seven circuit cards mounted on a common structural panel. One board includes the high voltage sweep generator, the main instrument interface circuitry, and the spacecraft interface connector. The other side of the mounting panel holds six high voltage converters with their interface mother-board. Two of these converters supply fixed high voltages to the sweep generator circuits. These connections are made by high voltage plugs and sockets mounted directly on the boards. The final component shown in the exploded view is the low voltage power converter mounted to a shield plate, which mounts above the high voltage supplies on metal posts. The electrical connections between the power converter and interface board are made with the only cable harness in the stack.

The stack subassemblies are mated by carefully sliding the two units together. The preamp boards have interface connectors that plug directly to mating connectors on the interface card. The anode plate high voltage plugs connect to sockets on either the interface board or the high voltage boards. The longer plugs pass through clearance holes in the interface board to reach the HV supplies.

The mechanical structure and enclosure of the stack is shared on one side with the electric field wire boom unit, which shares a vertical structure plate with the ESA stack. The back cover is a plate that includes support electronics for the wire boom unit. The second side is also a structural plate, and the top and bottom are made of thin aluminum sheets.

\subsection{ELECTRONICS DESIGN}

The electronic systems for each of the four ESA stacks are nearly identical and are summarized in the block diagram in Figure 7. They consist of four anode boards, two amplifier/counter boards, one board combining the main system interface functions and the HV sweep generator circuits, six high voltage converters, and the LV converter. The timing and programmable functions on these boards 


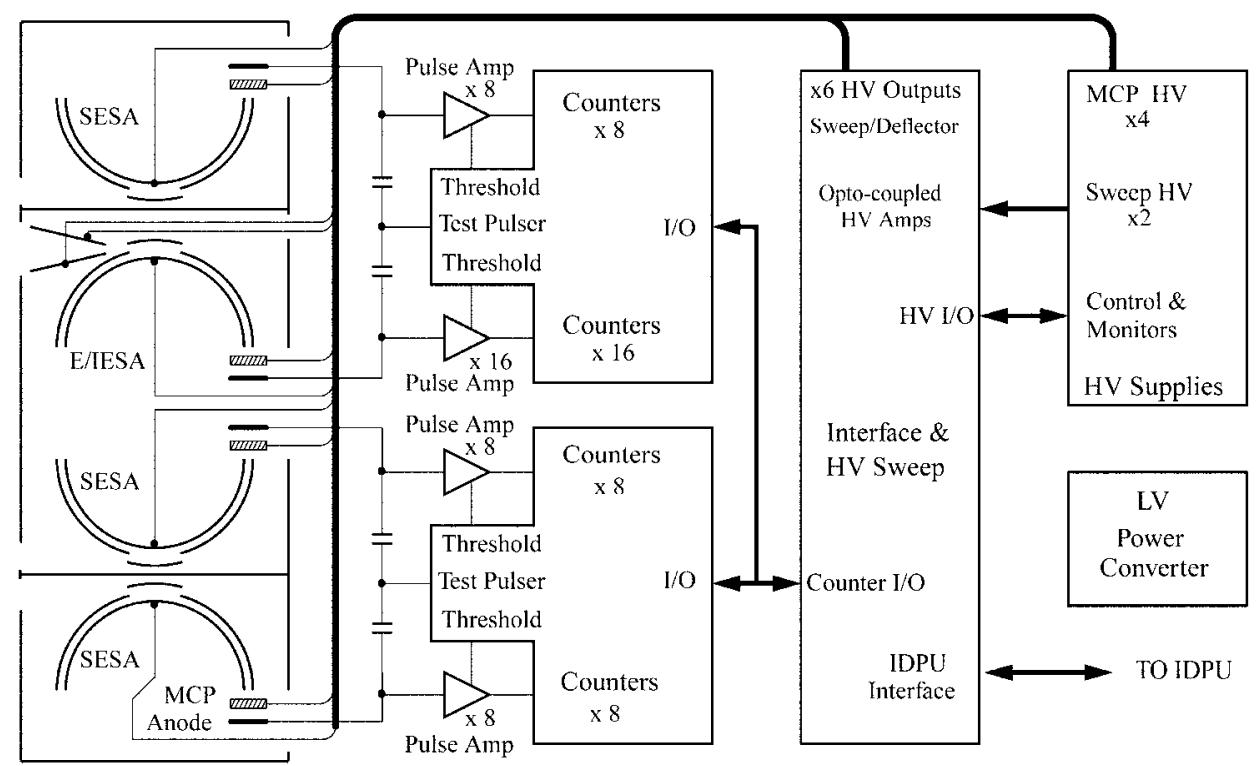

Figure 7. Block diagram of the electronics for a FAST ESA stack. The location of electrical component blocks are in the same relative positions as their mechanical counterparts in Figure 6.

are controlled by commands from the IDPU. All logic functions as well as certain analog functions are implemented in nine Actel A-1020 field programmable gate arrays (FPGA). These devices were an enabling technology for FAST. It is a single part type that is radiation resistant, consumes very little power, and replaces the functionality of approximately forty standard integrated circuits. The gate array can also be used for certain mixed digital/analog designs. All of the digital to analog converters in the FAST instruments are implemented by simply powering the FPGA with an accurate 5 Volt power source and driving standard R/2R resistor networks with the output logic gates.

The IDPU controls the operation of the ESA instrument with a serial I/O command interface. A spin-synchronous command gate recurring 12,288 pulses $\operatorname{spin}^{-1}$ serves as the master time base for the instrument measurement cycle. A 24-bit command data block containing 8 bits of address header and a 16-bit data word can be sent with each command gate pulse. The IDPU routinely issues 'broadcast' commands to all instruments every spin that contain spacecraft time, a 'spin sync' pulse once per spin, and 'magnetic phase' data that identify the phase angle between the spin sync and the location of the magnetic field plane. The commands for sweep and data format modes are also broadcast commands that go to all ESA stacks simultaneously. In addition to these critical timing and attitude data, the IDPU sends addressed commands to specific instruments. All configuration commands to the ESA stacks that control specific functions, such as the MCP voltage level for a specific sensor, are addressed commands. 
The data readout from the ESAs to the IDPU is a fixed format that is also synchronized to the spin. Each ESA stack has its own hardware interface to the IDPU, and data transfers are made at a clock rate of $1.048 \mathrm{Mhz}$ from all four stacks in parallel. Thus the data samples for a specific anode angle and energy from all four stacks arrive at the IDPU simultaneously and can be efficiently packed in a single data array. The IDPU reduces the 14 bit counter data to 8 bits via a pseudo square root compression.

Analog housekeeping signals are routed directly to the IDPU for digitization and formatting with other engineering data.

\subsubsection{MCP/Anode Board}

Some of the electrical functions of the anode board were already described in Section 2.3.3. The two main electrical design issues for the anodes are the high voltage power for the MCPs and the anode signal output coupling. The MCPs operate with a cross-plate potential of approximately $2 \mathrm{kV}$. The detailed high voltage configuration is different for the electron and ion sensors.

The ion MCPs operate with the input surface at high negative potential, which accelerates incident ions to improve their detection efficiency. The anodes operate at ground potential, and the output face of the MCP is biased at approximately $25 \mathrm{~V}$ negative to ground by a series resistor in its ground return. This slight acceleration voltage improves the focusing and collection of the output electron charge cloud by the anode. The electrical connection to the front and rear of all MCPs are bypassed with filter capacitors directly to the signal ground plane on the anode board.

The electron anodes operate with approximately $600 \mathrm{~V}$ of pre-acceleration voltage to ensure good detection efficiency for low energy electrons. A series resistor could generate this voltage, but it would dissipate approximately $25 \%$ of the MCP power. The FAST design conserves this power by tapping the pre-acceleraton voltage from the first stage of a five stage voltage multiplier in the HV supply. The output electron cloud is post-accelerated by about $25 \mathrm{~V}$ by an MCP series resistor. Because the electron anodes operate at high voltage, the output signal is coupled through monolithic ceramic capacitors that are surface mounted to the anode with silver epoxy.

Two anode versions are used. The board for the EESA and IESA analyzers uses 16 anode pads to cover the $180^{\circ}$ annulus. The component loading is different for these two versions, but the same anode design is used. The SESA anode has 8 anodes, each with $22.5^{\circ}$ angular width. The MCPs and their mounting hardware are identical on all anode types.

The anode electrical design resists crosstalk and noise pickup by the anode electrodes. An internal ground-plane shields the anode pads from each other and from other signals on the back surface of the board. Alternate pins on the signal connector joining the anode board with the preamplifier circuit board are also grounded. No crosstalk between anodes pads was found over the entire range of MCP pulse amplitudes. The design is also resistant to external noise pickup. A 
small amount of duplicate event counting is introduced at the boundary of anodes, where the electron cloud from the MCP spreads to anode pads on each side of the boundary. The gaps between the anode pads and the gap between the MCP and the anode surfaces are much smaller the anode width, so this double counting contributes only a few percent to the total rate.

\subsubsection{Amplifier/Counter Boards}

The MCP output signals are amplified, discriminated, and accumulated by circuitry on the counter boards. The pulse amplifiers are the AMPTEK model A121, with their dead-times adjusted to $140 \mathrm{~ns}$. These amplifiers have an adjustable threshold that can be set by ground command. The threshold can be adjusted for optimum noise rejection, and is also used to measure pulse height distributions of the MCPs on orbit. A diagnostic function included on this board is a programmable tail pulser that is used for in-flight testing of the preamps.

Two versions of the counter board were required, since the two SESA analyzers are identical with 8 anodes each, while the second pair of analyzers has 16 anodes on the I/EESA analyzers. All digital functions are implemented in Actel A-1020 programmable gate arrays. Three configurations of the gate array are used on the counter boards. The basic functions performed by these circuits include the following:

(1) A Decoder for commands received from the IDPU.

(2) A 14 bit counter, latch and readout multiplexer for each of 8 anode channels. Also includes a programmable input mask to disable noisy anode channels.

(3) A Despun channel selector to send raw counts from any addressed pitchangle channel to the IDPU (used for the wave/particle correlator).

(4) A Despun channel selector to send raw counts from the channel aligned with magnetic field direction (used for real-time energy flux computation).

(5) A D/A converter to set the amplitude for a test pulser; also a tail-pulse generator switch.

These functions are implemented in three specific FPGA circuit designs. The first circuit is the master device that includes the command decoder, the output data interface, an 8 channel counter circuit, and the data selector for pitch-angle selected counts. The second FPGA contains an 8 channel counter circuit, the data selector for magnetic field-aligned counts, and the test pulser composed of a D/A converter and switch. These two gate arrays are used on both versions of the counter board. A third FPGA design is used only for the I/EESA board that contains the extra 8 channel counter circuit.

The block diagram shows the test pulser capacitively coupled to the preamp in parallel with the anode input. The pulser signal line is simply routed perpendicular to the input traces on the preamp board, with the trace widths selected to provide a fraction of a picofarad of coupling. The threshold control voltages for the preamplifiers are generated by A/D converters on the main interface board and routed to the counter boards. 
The A121 preamps are hybrid in-line packages that are mounted vertically on the circuit board as shown in Figure 5. An aluminum channel runs across the top of each row of amplifiers for radiation shielding and vibration support. It was attached to the amplifiers with staking material and anchored to the pbc with standoff posts.

The preamp board plugs directly onto connectors on both anode boards in the analyzer set, and is mechanically mounted to the structural plate on the analyzer. Each preamp board plugs directly to the main interface board with a board-mounted connector.

\subsubsection{Interface/HV Sweep Generator Board}

The interface and HV sweep functions are relatively independent functions that are combined on a single circuit card for the convenience of their interconnection with the other instrument electronics. The interface circuit is the direct connection between the instrument and the IDPU. It decodes and generates the counter load commands for the preamp boards, and consolidates the serial output telemetry data stream. It also controls the analog multiplexer for the housekeeping data that are sent to the IDPU. A special data product computed by the interface circuit is the electron energy flux. This quantity is derived from the despun field-aligned EESA anode, and is a real-time estimate of the energy flux within a selected energy range that is used by the IDPU trigger algorithms to determine mode changes and burst collection.

The other major function of the interface circuit is to generate a number of analog voltages used to control the high voltage converters and the threshold settings of the MCP pulse amplifiers. All four of the FPGA circuits for the interface and HV control functions have an accurately regulated $5 \mathrm{~V}$ supply so they can drive resistor networks for D/A conversion. These circuits generate the six reference voltages that control the high voltage converters and the four threshold control voltages for the MCP pulse amplifiers.

The high voltage sweep generation circuits include waveform synthesis logic and D/A conversion that are contained in two FPGAs, and six independent high voltage drive circuits that use opto-coupled push-pull output stages. The optocouplers are the AMPTEK HV601. The high output capability of these amplifiers produces a full-scale settling time of about $1 \mathrm{~ms}$ for a $4 \mathrm{kV}$ step. The driver circuits for the opto-couplers have a high peak output current capacity that exceeds their continuous dissipation limit. A protection circuit verifies that the raw high voltage supply is greater than a minimum level that will guarantee closed-loop operation of the sweep circuit. IDPU software also checks that the voltage of the fixed HV supply is adequate for the programmed sweep levels.

The high voltage waveform synthesis can generate a total of 174 distinct values. The typical 48 level spectrum is determined by the detector accumulation interval. During each data accumulation the sweep high voltage supply is stepping through 4 micro-step levels. At very low voltages the step resolution only allows one or two micro-steps in each accumulation. The micro-steps are used to approximate a 
smooth voltage sweep rather than distinct steps. The digital codes for these levels are computed with FPGA hardware logic. Similar synthesis logic is used for both the continuous sweep used by the ESAs and the more complicated sweeps possible with the SESA.

Three of the high voltage outputs serve the SESA sensors, and the other three are used by the I/EESA analyzer and its two deflectors. The deflectors only produce positive deflections to avoid photo-emission, so one of the two deflectors is always at ground potential and the other is sweeping. The deflector voltage is computed to be a fixed fraction of the analyzer voltage step, depending on the desired deflection angle. The angle can be selected from 8 angle levels that are approximately $1.2^{\circ}$ per step.

\subsubsection{HV and LV Supplies}

Four power converters supply high voltage to the MCPs, and two additional converters supply the fixed high voltage needed by the sweep generator circuits. All supplies use a sine-wave power oscillator driving a voltage multiplier stack and operate at a frequency of $60 \mathrm{kHz}$. The nominal maximum voltages are $3 \mathrm{kV}$ for the MCP bias supply voltages and $5 \mathrm{kV}$ for the sweep supply. All converters are voltage programmed and have output current and voltage monitors. Their input supply voltage is $28 \mathrm{~V}$.

The low voltage converters for the stacks are sine-wave converters operating at $60 \mathrm{kHz}$ operate from a regulated $28 \mathrm{~V}$ input. There is no output regulation. The primary regulation is supplied by the central power system in the IDPU box.

\subsection{TESTING AND CALIBRATION}

\subsubsection{Component Testing}

The first level of component testing included qualification of new component types. All electronic components for FAST had to be qualified to a radiation level of $30 \mathrm{kRad}$ or greater. A number of new components that did not have radiation specifications were tested for FAST at the Goddard Space Flight Center (GSFC) test facility. The AMPTEK A121 pulse amplifier the AMPTEK HV601 high voltage opto-coupler were key components for the plasma instrument that did not have radiation data. They were tested and found fully functional after a dose of $100 \mathrm{kRad}$. The Actel 1020A programmable gate array (FPGA) was used to implement virtually all of the digital logic for the FAST science instruments. This component type had previous test data, and lot testing of the FAST parts found them functional up to $100 \mathrm{kRad}$.

Functional testing and matching were performed on several key components and subassemblies before installation in circuit boards. All A121 pulse amplifiers were individually tested to determine threshold and gain uniformity, noise susceptibility, and variation of pulse width and dead time with pulse amplitude. The Actel FPGAs were all verified in breadboards prior to assembly. 
Microchannel plates underwent an extensive process of preparation and testing. They were inspected, given an ultrasonic alcohol scrub, and baked, prior to vacuum testing. Pairs of plates were initially matched according to manufacturers data on plate resistance and gain, and then were evaluated in vacuum chamber tests. Bellshaped pulse height distributions with a FWHM of less than $100 \%$ were obtained at an electron gain of $\sim 10^{6}$ for all flight plates. After final selection, MCPs were installed in their flight anode boards, re-tested, and stored in $\mathrm{N}_{2}$ dry boxes. A total of 20 anode board assemblies (16 flight and 4 flight spares) were assembled and tested.

The analyzer assemblies followed similar test and inspection procedures. Analyzer hemispheres were visually inspected after gold-blacking to check coating uniformity and remove any dust or other contaminants. The analyzer mechanical assemblies underwent preliminary vacuum chamber testing to determine their energy-angle response, azimuthal uniformity, and UV rejection, and to identify any high voltage arcing or other problems. Upon completion of these tests the analyzers were joined with the tested MCP/anode assemblies and the detector electronics boards. The analyzer units were then ready for calibration.

\subsubsection{Calibration}

Calibration and verification of the ESA sensors were performed in three phases. First, a complete calibration and thermal vacuum test was performed at UC Berkeley prior to delivery to GSFC for spacecraft level integration and testing. Second, during the spacecraft thermal vacuum test at GSFC, the analyzer covers were opened and the instruments were run during flight simulations, and stimulated by electron and ion guns and radioactive sources. After completion of the spacecraft integration, the instruments were returned to UCB for inspection and refurbishment. MCPs are sensitive to contamination so there was concern about contamination occurring during the spacecraft tests. The analyzers were opened up for inspection and tested for any change of gain or other symptoms of contamination. No evidence of contamination was found, so the detectors were reassembled for a complete re-calibration and short thermal vacuum test. The entire procedure was completed for all 16 detectors during a two week period. The modular detector design made it possible to disassemble a four analyzer instrument stack down to the individual component level in about four hours. The automated calibration procedure performed a complete angle/energy calibration of an instrument stack in less than one day. Our approach to serviceability and automated testing was fortunate, since launch vehicle delays forced us to repeat this inspection and calibration procedure two more times during the subsequent two years until launch.

The automated ESA calibration used a computer to control a three axis manipulator and high voltage particle accelerators, and also commanded an interactive GSE program on a second computer that controlled the instrument. The electron gun is a photoelectron source that uses a back-illuminated quartz window with a semi-transparent chromium cathode. A grounded planar grid in front of the cathode 
produces a uniform, parallel electron beam with energies up to $30 \mathrm{keV}$ over a diameter of $10 \mathrm{~cm}$. Helmholtz coils surrounding the chamber were used to null the Earth's field for low energy testing. The ion gun uses an electron impact ionization source. The manipulator has three degrees of freedom: a rotisserie for rotating the sensor around the pitch-angle axis, a yaw axis to change the particle elevation angle, and a linear translation axis that allowed consecutive testing of all four analyzers in the instrument stack. The GSE computer contained an interface card that duplicated the IDPU interface and allowed the computer to configure and read data from the ESA stack. The GSE computer logged all calibration data during the tests.

The ESA calibration procedure included six basic tests:

(1) A complete array of analyzer voltage versus elevation angle data was collected at azimuth angles of $-80^{\circ}, 0^{\circ}$ and $+80^{\circ}$ for two separate gun energies to determine the analyzer constant (energy/voltage ratio) and verify a uniform energy/angle response.

(2) Hemisphere concentricity was tested by performing an energy sweep at the central elevation angle for each $5^{\circ}$ azimuth angle step to verify that there was no shift of the detected energy peak with angle.

(3) The optimum MCP bias voltage was found by stepping the programmable threshold of the A121 amplifier to measure the integrated pulse height distribution of the MCP for several values of high voltage bias. This test was made for several values of azimuth angle to map the gain uniformity at different positions on the MCP. This test is also run periodically during flight to monitor MCP gain changes.

(4) Verification of the programmable sweep voltage was made by setting the gun to the nominal ESA energy levels and stepping the hemisphere voltage through equivalent voltage steps.

(5) The relative geometric factor of the anodes within each electron sensor was determined using a $\mathrm{Ni}^{63}$ beta source to illuminate the analyzer while the sensor was rotated azimuthally.

(6) The sensor was configured to operate in several flight modes, with high voltage sweeping, while the gun was adjusted to verify that counts were in the proper energy bins.

Individual test runs required between 15 and $60 \mathrm{~min}$ to complete. Results from each test were immediately plotted and checked during the subsequent run so any anomalous results could be rechecked.

Absolute geometric factors for the analyzers were estimated from computer simulations, the known grid transparencies, and published values of MCP efficiency. Calibration data from the $\mathrm{Ni}^{63}$ beta source test showed $<20 \%$ variations in geometric factor around the analyzer azimuth field of view. Sensors were also tested for in-flight uniformity, using relatively isotropic fluxes of plasmasheet particles. Small corrections in sensitivity based on consistent variations in these measurements have been included in data analysis calibrations. The absolute geometric factor for the electron ESAs were tested in-flight by comparing measured magnetic 
deflection while crossing inverted $\mathrm{V}$ arcs with the expected deflection due to current carried by the measured electron flux. These comparisons showed remarkably good agreement (see McFadden et al., 1998b, Figure 1). The electron sensor geometric factor was also checked by comparing the density determined by the ESA data with the absolute density estimated from measured plasma wave cutoffs (Strangeway et al., 1998).

The ion sensor absolute calibration was determined through cross calibration with the electron sensor in the auroral acceleration region. This cross calibration was performed in regions where the energetic $(>100 \mathrm{eV})$ electron and energetic $(>30 \mathrm{eV})$ ion densities dominated the total density. Selecting a region where one could neglect any cold electron component was necessary since photoelectrons produced by the spacecraft and secondary electrons produced within the ESA analyzer can contaminate the low-energy electron measurement. Events were picked where densities could be compared with measured plasma wave cutoffs. The neglect of any low energy ions introduced a small $(\sim 20 \%)$ correction due to scattered energetic electrons contaminating the low energy ion measurement. This latter assumption was justified by comparison with the low energy ion measurements made by the TEAMS mass spectrometer which was not susceptible to this contamination. In addition, the mass spectrometer data were used to correct for ion composition, although this correction was small because the selected data set had a density dominated by $\mathrm{H}^{+}$. The ion sensor absolute geometric factor was subsequently reduced by $35 \%$ from the estimated pre-flight value in order to obtain agreement between the measured densities. These ion and electron relative geometric factors were subsequently verified on 10 other orbits containing ion beams (McFadden et al., 1999).

The on-orbit performance of the ESA sensors has been exceptional. No radiation damage is evident in the electronics after 3 years of operation. MCP high voltages were increased only during the first year, typically by $100 \mathrm{~V}$, to correct for expected gain decreases during early aging of MCPs. The detectors continue to function normally with low background rates. A discussion of in-flight background rates, including penetrating radiation, solar UV, spacecraft photoelectrons and scattered electrons are discussed by McFadden et al. (1999). In summary, the uniformity and stability of the ESA sensors and their ability to accurately obtain absolute measurements of particle fluxes, densities and currents have exceeded expectations.

\subsection{IDPU FUNCTIONS AND OPERATING MODES}

The IDPU provides command and control of all the instruments on FAST. Since the FAST instruments are shut down each orbit to conserve power, the IDPU must perform a complete initialization of these sensors for each orbit. It was desired that this initialization should be flexible enough to change from orbit to orbit, or even within an orbit. The command load required to accomplish this task was much too large 
to be uploaded from the ground. Instead, a set of operating modes were developed which would be kept onboard and called out by single commands. This approach reduced the instrument command load to fewer than a dozen commands per orbit, which was compatible with the spacecraft command memory. This small command set includes the particle sensor and field instrument operational mode commands, burst memory trigger commands, fast survey memory trigger commands, and fast survey memory allocation commands. The operational mode command contains a two byte code that selects one of eight stored 'particle mode' commands and one of eight 'field mode' commands. The particle mode configures the ESA and TEAMS instruments and includes command subsets for switching between 'fast survey' and 'slow survey' data collection. Detailed descriptions of instrument commanding are given by Harvey et al. (this issue) and McFadden et al. (this issue).

The particle mode is used to configure the ESA instrument, including powering low voltage supplies, initializing low voltage electronics, ramping up high voltages, monitoring the currents, setting high voltage sweep waveforms, and initializing the data collection and averaging hardware that generates burst and survey data. One of the eight particle mode commands is reserved for shutting down the ESAs in an orderly sequence at the end of each pass. The particle modes consist of a relatively small command set ( $<30$ commands), but includes some general commands that initiate macro command sets that are common to all the particle modes. Two examples of the macro commands are the 'low voltage on' command which initiates a sequence of $\sim 50$ commands that initializes the low voltage power supplies and sets instrument control registers, and the 'high voltage on' command that ramps up all the high voltages to their nominal values. Furthermore, these macro commands are controlled by a programmable table which contains default values for all high voltage and low voltage registers. Additional commands in the particle mode are used to control data sampling times and high voltage sweep waveforms as described below. The FAST command strategy provides a relatively flexible but simple method to control the instruments.

The use of various particle modes is primarily to adjust data sampling rates and high voltage sweep waveforms. These functions are controlled through $4 \mathrm{com}-$ mands: ACCUM, IMODE, EMODE, and SMODE. The ACCUM command controls the data collection time within the ESA, with choices of $1.63,3.3$, or $6.5 \mathrm{~ms}$ accumulations. All data collection has been at the highest setting $(1.63 \mathrm{~ms})$ since launch and other accumulation times will only be used as a backup if reduced data rates are required.

The IMODE and EMODE commands allow selection of various energy sweeps for the EESA and IESA pairs. Since no instrument degradation has been observed and no science consideration has indicated a need to change this mode, these instruments continue to operate in the normal mode with a 48 step sweep over the full dynamic range each $78 \mathrm{~ms}$. However, the IMODE and EMODE commands allow adjustment of the highest energy sampled or can be set to produce 96 energy step mode with half the time resolution. In case of failure of the EESA, backup 
commands are available to swap the EESA pair with one of the SESA pairs. The SMODE commands configure the six analyzer pairs that make up the electron spectrograph measurement. The SESAs can be configured into four operating modes, (FIXED, STEPPED, TRACKING, and SWEEPING) discussed below.

The SESA FIXED mode provides the highest time resolution continuous monitor of the pitch angle distribution at 6 energies. This mode is primarily used to monitor electron flux modulations associated with ion cyclotron waves and solitary waves, which are resolved at frequencies up to $\sim 300 \mathrm{~Hz}$ (see McFadden et al., 1998b). Factor of 2 separation between energy levels is normally chosen in this mode to provide a wide enough energy range so that samples near the energy flux peak are obtained. For nightside aurora, a 0.5 to $16 \mathrm{keV}$ energy range is normally selected and for dayside aurora the range is reduced to 0.12 to $4 \mathrm{keV}$.

The SESA STEPPED mode was devised to increase the energy resolution of the measurement at the expense of time resolution. Each SESA pair is stepped through 2,4 or 8 energy steps, providing a 12, 24 or 48 step energy measurement. The 12 energy sample mode was initially expected to be the best compromise for the SESA since it could provide a wide enough energy range with adequate $(\mathrm{d} E / E \sim 1.4)$ energy resolution to resolve most features of the distribution. However, it was found that its sampling rate was often inadequate for electron modulations and that its energy resolution was not satisfactory for resolving changes in spectra associated with electrostatic shocks. The 4 and 8 energy step modes have proven to be the most useful for resolving the electron distributions across small scale $(\sim 100 \mathrm{~ms})$ electrostatic shocks, providing evidence that these are static structures and not temporal variations in the electric field.

The SESA TRACKING mode was designed to provide a high time and energy resolution measurement of the auroral electron beam. It was known from sounding rocket measurements that higher frequency electron modulations are normally only observed on a steep slope of the distribution above the energy flux peak. Although the FIXED mode with appropriate choice of energy levels normally provides a sample on this steep slope, typically only a single energy level measures statistically significant modulations. The TRACKING mode provides multiple energy samples of electron modulations by selecting 6 closely spaced energy levels that are determined from the electron energy spectra. The IDPU uses measurements from the EESA to determine the energy with the peak count rate. The IDPU then commands the SESAs to a set of energy steps relative to this energy, with levels normally chosen to straddle the energy flux peak. This mode has been useful in measuring small rapid shifts in the spectral peak, which produce out-of-phase changes in count rate above and below the spectral peak, and thus demonstrating that these are modulations of the accelerating electric fields and not modulations of the input electron flux.

The SESA SWEEPING mode was devised to provide a higher time resolution measurement that was identical the EESA measurement. All six SESA pairs are swept over the entire energy range, but their sweeps are phased relative to each 
other so that the the combined measurement has 6 times the time resolution of the EESA. This mode produces a measurement similar to the STEPPED mode with 48 energies, but reduces problems associated with the high voltage slew rate to a single energy sample. This mode is also useful for cross calibration since all sensors perform the same measurement.

\section{Initial Science Results}

The high resolution plasma experiment on the FAST satellite has performed as intended and has yielded a wealth of new scientific results. The initial results can be found in the special FAST section of Geophysical Research Letters (1998, Vol. 25, number 12). Some highlights of these results include the observation that accelerated ionospheric electrons are a major source of the downward auroral currents, the quantitative demonstration that auroral acceleration is consistent with quasi-static potential acceleration in both the upward and downward current regions, the identification of electromagnetic ion cyclotron (EMIC) wave acceleration as a major modification to the quasi-static potential acceleration, the measurement of electron modulations by electron solitary waves, demonstration that the auroral density cavity is devoid of cold plasma, and conclusive evidence that electron 'horse-shoe' velocity distributions are the energy source for auroral kilometric radiation (AKR). Most of these new observations can be attributed to the design approach outlined in this paper, which combined high energy, angle and time resolution measurements of electron and ion pitch angle distributions with onboard triggers to capture the highest quality observations.

Figure 8 presents data from a nightside auroral crossing that illustrates the range of energy, angle, and time scales covered by the plasma instrument. The spectrograms have not been smoothed so that the measured time resolution can be fully appreciated. The upper two panels show about $5 \mathrm{~min}$ of electron data out of a $\sim 35$ min data set collected across the northern auroral zone. Observations prior to 10:13 UT were made in a 'fast survey' mode with $312 \mathrm{~ms}$ time resolution, whereas the later observations were in 'slow survey' mode with the $2.5 \mathrm{~s}$ resolution. The survey mode transition at 10:13 was initiated by the onboard data collection routines to prevent memory overflow.

Several different auroral phenomena can be discerned from the survey data. The top panel shows two large inverted $V$ arcs (10:10:55-10:12:18 and 10:12:30 $-10: 13: 30)$ that comprise the bulk of the region one upward current system. The dropout in precipitation between them corresponds to a downward current region. The brief appearance of low energy $(\sim 10 \mathrm{eV})$ upgoing field-aligned electrons, identified by their $180^{\circ}$ pitch angle in panel 2 , indicates that FAST was crossing near the lower edge of the downward current acceleration region. A much clearer example of accelerated electrons carrying a downward current is seen between 10:10:30 and 10:10:55, where a series of short duration sheets of upgoing elec- 


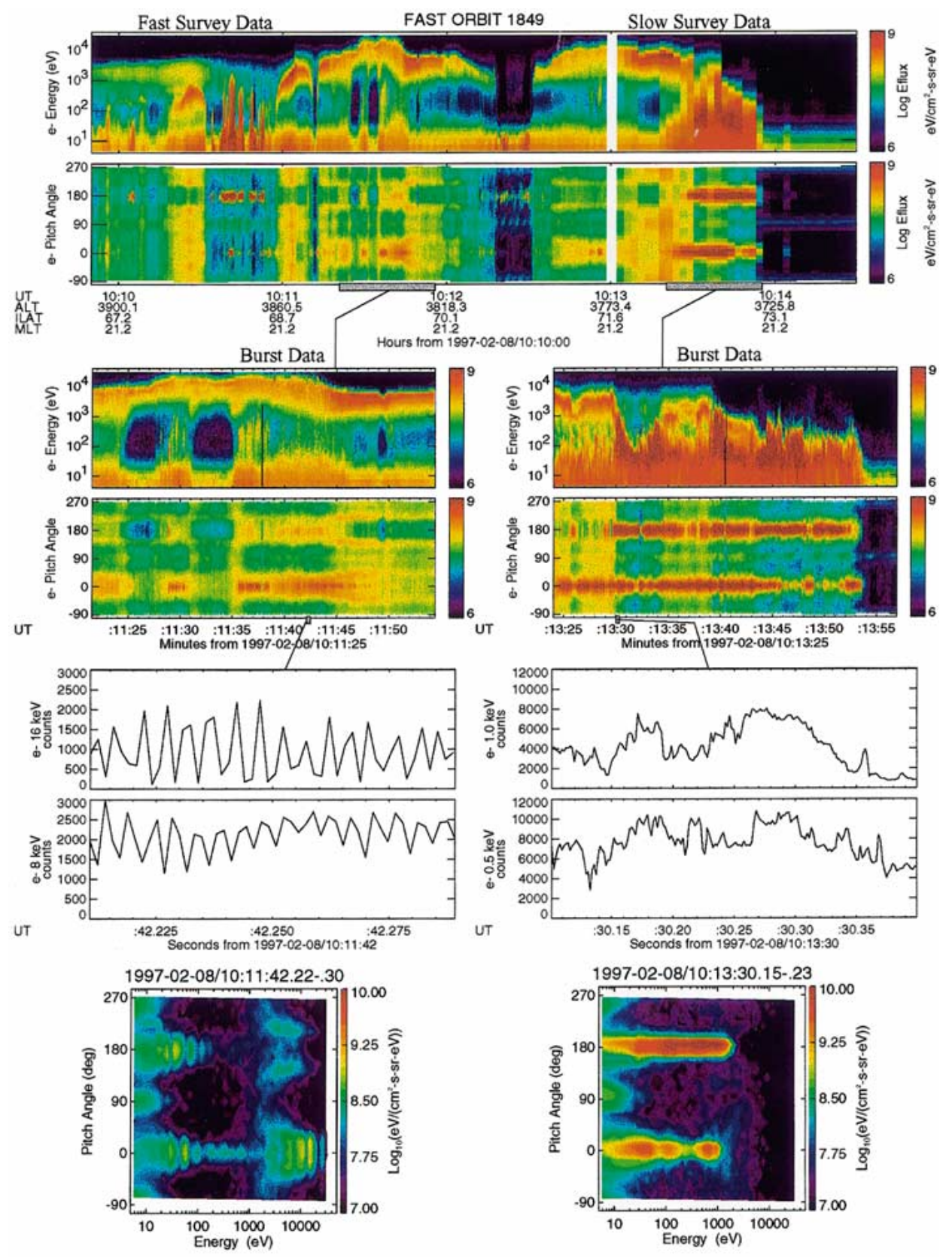

Figure 8. Electron measurements by the FAST satellite during a crossing of the nightside auroral zone. The top two panels show electron energy and pitch angle spectrograms with 'fast survey' and 'slow survey' data appearing before and after 10:13 UT, respectively. Panels 3 and 4 show burst data captured by the onboard triggering system that was keyed to the measured electron energy flux. Panels 5 and 6 show burst data from fixed energy channels of the SESA detectors that show modulations on the $1.6 \mathrm{~ms}$ sampling time scale. The bottom two plots are energy-angle distributions of energy flux measured by the EESA illustrating intense field-aligned beams. 

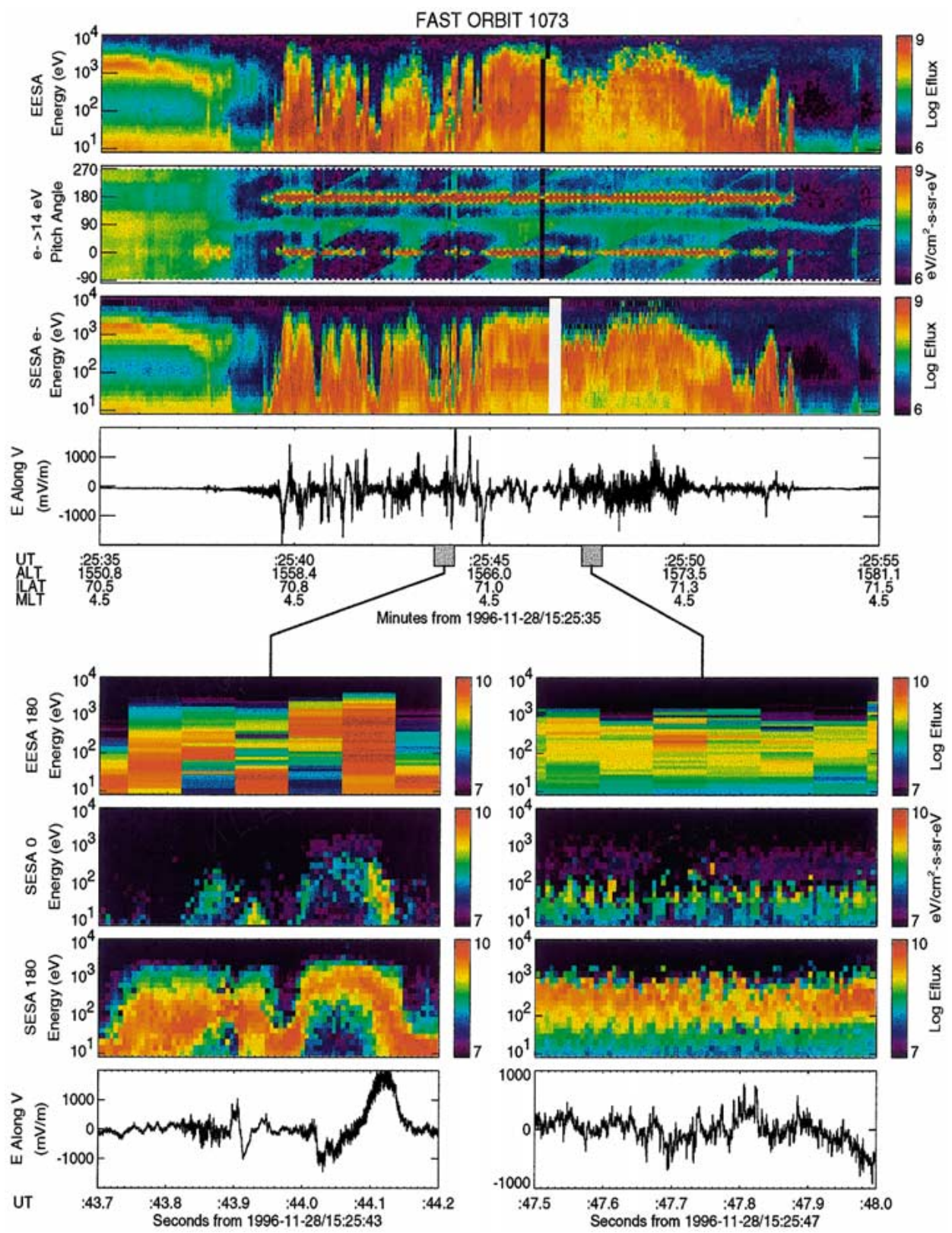

Figure 9. Electron measurements by FAST near the pre-midnight polar cap boundary. These data illustrate some of the physical processes that can be resolved by the high time resolution measurements of the SESA instrument. 

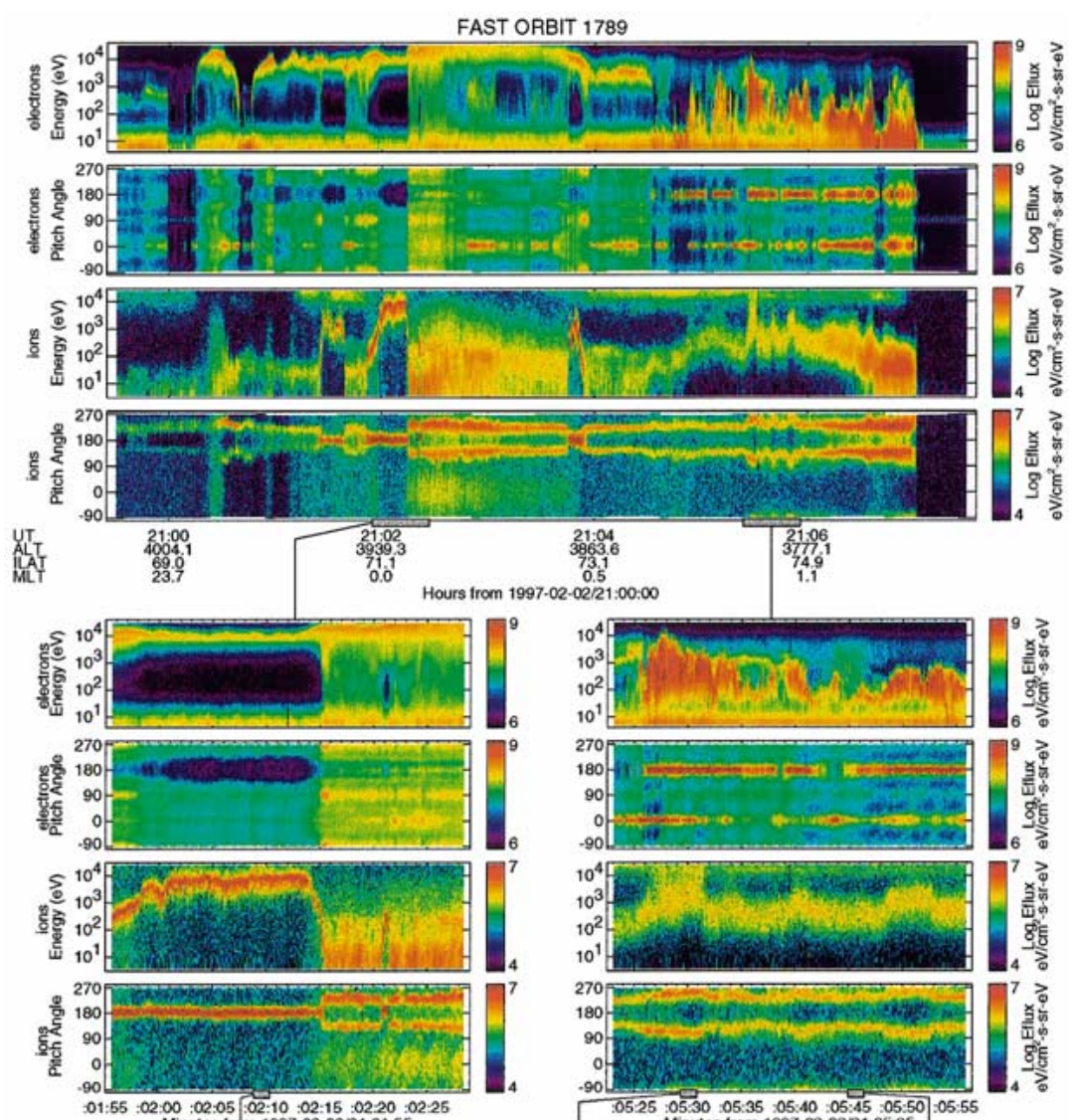

$01: 55: 02: 00: 02: 05: 02: 10: 02: 15: 02: 20: 02: 25$

21:02:10
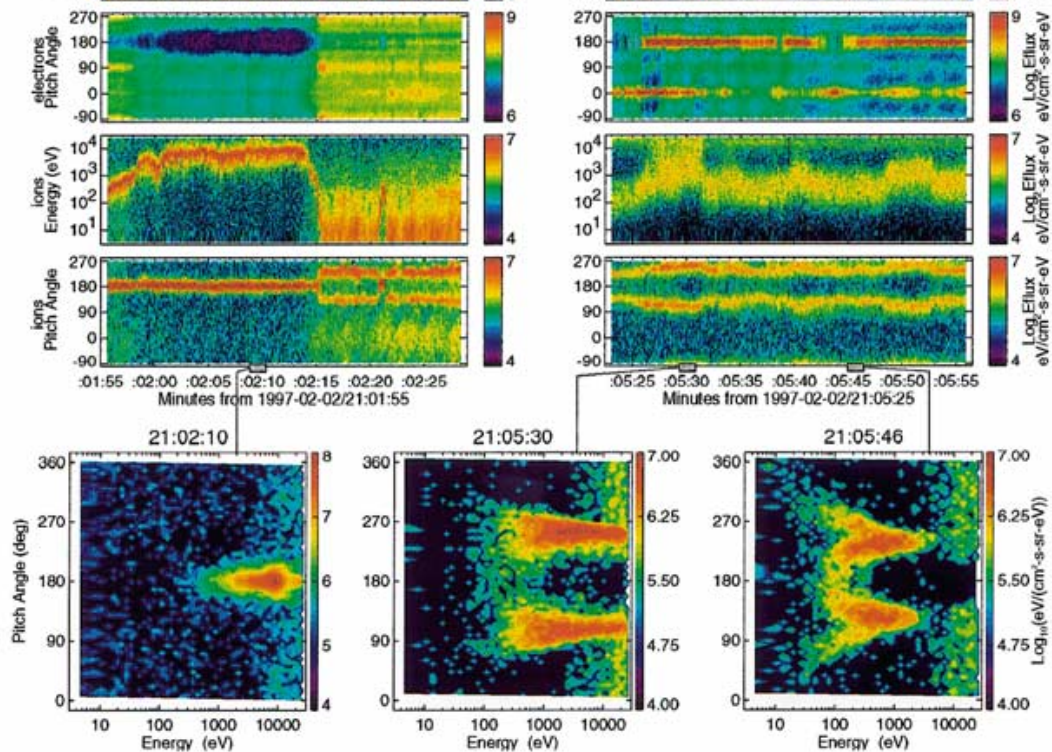

90 L 2.

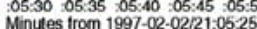

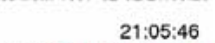

Figure 10. Electron and ion measurements from the FAST satellite during a pre-midnight auroral zone crossing. The configuration of the FAST instruments allow continuous measurements of ion beams and ion conics on time scales much faster than the spacecraft spin period.

trons are observed. The small scale spatial structure of these electrons, coupled with their extremely narrow $\left(\sim 1^{\circ}\right)$ field-aligned pitch angles, made these particles difficult to observe on previous auroral satellites. FAST's continuous monitoring of electron fluxes both upward and downward along the magnetic field, coupled with its high time resolution, has allowed it to observe these electrons on most orbits 
with apogee in the winter hemisphere indicating they are a common feature that was not obvious prior to FAST.

Two burst captures were triggered during this pass that are displayed with expanded time scales in panels 3 and 4 . Their location and duration are identified by the shaded bars at the bottom of panel 2. The burst data reveal structure not resolved in the survey data, such as the short duration bursts of field aligned electrons at energies visible below the inverted V spectral peak in the first burst. These field aligned electrons are cold electrons that have been accelerated by EMIC waves. Rapid modulations of the electron distribution caused by these waves were captured by the 8 and $16 \mathrm{keV}$ fixed energy channels of the SESA as shown in the expanded time scale panels 5 and 6 (left side). These $1.6 \mathrm{~ms}$ measurement samples are just able to resolve the $\sim 220 \mathrm{~Hz}$ modulations that occur just below the $\mathrm{H}^{+}$ cyclotron frequency. These modulations also appear in the energy-angle distributions where they cause intensity modulations at different energies during the energy sweep (left side, bottom panel). The most intense modulations by EMIC waves are observed within inverted $\mathrm{V}$ arcs that include a lower temperature, magnetic field-aligned electron population (left side, panels 4 and 7).

Another feature often seen in the FAST data is counterstreaming electrons at the polar cap boundary near midnight (10:13:25-10:13:55 UT). The large electron flux in this region triggered burst data capture of the event as seen in panels 3 and 4 (right side). These EESA burst data, with $78 \mathrm{~ms}$ time resolution, capture a wealth of structure in the electrons completely missing from the survey data. However, even these high resolution data fail to capture all the structure. Panels 5 and 6 (right side) show the $1.6 \mathrm{~ms}$ time resolution data from two fixed energy channels of the SESA. Structure in the precipitation is seen down to the smallest time scales. This structure will often reveal itself in the distribution function measurements (right side, bottom panel) as time aliasing in the detector energy sweep. The bottom panel displays a counterstreaming electron distribution illustrating the broad energy range over which this precipitation is observed. The pitch angle width of the beam in the plot is limited by the detector resolution $\left(11.2^{\circ}\right)$. However, the use of fixed energy SESAs has allowed an estimate of the beam width by examining the rise in count rate as fixed energy detectors rotate through these beams. These measurements establish that the beams are extremely narrow $\left(\sim 1^{\circ}\right)$ with perpendicular temperatures comparable to the ionospheric thermal population.

The counterstreaming and upgoing electron beams often contain rapid time variations in their energy structure that are best resolved by the SESA cycle mode. Figure 9 shows another example of the polar cap boundary region. The top two panels present a 20 second burst capture of electron energy flux and pitch-angle spectra collected by the EESA, with $78 \mathrm{~ms}$ time resolution. These data have resolution comparable to the burst example in panels 3 and 4 of Figure 8 . The third panel displays SESA burst data with 24 energy levels and $6.5 \mathrm{~ms}$ time samples that resolve time structure missing in the EESA data. The character of the electron fine structure changed about half-way through this event, which is also evident 
in the electric field data plotted in panel 4. The first half of the event contains structured upgoing electron beams that are correlated with large bipolar electric field structures. These field signatures are consistent with quasi-static U-shaped potential structures that accelerate the electron beams. The second half of the event contains higher frequency electric field variations and fine-scale modulation of the electrons that result from wave-particle interactions in the upgoing electron beam.

The two sets of panels in the lower half of Figure 9 are expanded plots of 0.5 second intervals from these two regions. Panel 5 displays the upgoing $\left(180^{\circ}\right)$ magnetic-field-aligned electron flux measured by the EESA, which can be compared with the upgoing $\left(180^{\circ}\right)$ and downgoing $\left(0^{\circ}\right)$ fluxes measured by the SESA shown in panels 6 and 7. The panels on the left show a cut across two distinct accelerated beams. The second beam, which is crossed in about $0.1 \mathrm{~s}$ starting at 15:25:44.0, is the more energetic and distinct event. The bipolar electric field signature in the bottom panel has an amplitude greater than 1 volt $\mathrm{m}^{-1}$. The EESA measurement barely resolves this structure, whereas it is resolved in detail by the SESA. Assuming that this beam is purely a spatial feature, it maps to a $0.6 \mathrm{~km}$ width at ionospheric heights, and the individual data samples represent a $40 \mathrm{~m}$ scale size. The right-hand set of panels 5-8 show a different signature of electron energy variation, which is the rapid energy modulation of the upgoing electron beam. In this case, the only evidence recorded by the EESA is the aliased modulation during the energy sweep. The SESA data show a quasi-periodic modulation of the electron energy by several hundred volts at approximately $30 \mathrm{~Hz}$. The electric field contains intense broad-band low frequency waves with an amplitude of several hundred $\mathrm{mV} / \mathrm{m}$. The electron modulation is apparently caused by interaction with these intense ion cyclotron and fast solitary waves.

Figure 10 highlights some of the ion measurements made on a typical midnight sector auroral pass. The top four panels shown 8 minutes of electron and ion fast survey data near the high latitude boundary of the auroral zone during a moderately active period ( $\mathrm{AE} \sim 500)$. The electron measurements in the first two panels show a signature similar to the event in Figure 8. This includes a wide region of inverted V electron acceleration between 21:00:00 and 21:04:30 UT, followed by an interval of upgoing and counterstreaming electron beams near the polar cap boundary. Panels 3 and 4 show typical ion properties of this region. Three ion beams are easily identified in panel 3 by their intense energy flux and narrow energy spread. The pitch angle distributions in panel 4 show that the beams are closely aligned near $180^{\circ}$.

Conical ion distributions are evident by their pitch-angle signature, which lies near the loss cone angle on both sides of $180^{\circ}$. Conics within the inverted $\mathrm{V}$ have broad energy spectra typically ranging from a few $\mathrm{eV}$ to a few hundred $\mathrm{eV}$. The ion conic signature is distinctly different in the region of upgoing electron beams near the polar cap boundary. Here the ion conics are generally more energetic, with spikes of high energy coinciding with the most energetic electron beams. The most 
intense, although less energetic conic fluxes are found near the polar cap boundary, coincident with the intense counterstreaming electron fluxes.

The left set of burst panels (5-8) display a high resolution view of the ion beams and conics found in the inverted $\mathrm{V}$ regions. Panels 7 and 8 demonstrate the ion beam energy variations and the sharp boundaries typical of ion beams. A low energy ion beam lasting only a fraction of a second is fully resolved in the ion energy flux and pitch angle plots at 21:02:21 UT. The ion beams are created when a portion of the inverted $\mathrm{V}$ electrical potential extends below the altitude of the spacecraft and accelerates ionospheric ions upward. The potential above the spacecraft decreases by the same amount, which is apparent from the slight decrease of the electron beam energy coincident with the ion beams. The potential below the spacecraft also suppresses ionospheric thermal and secondary electrons and produces a density cavity within the ion beam, as is evident in panel 5 . The complete depletion of the electron loss cone is also clear from panel 6. A typical pitch angle distribution for the beam ions is found in the bottom left panel. The fine-scale structure within the beam results from slight differences between energy distributions for each of the ion species that arises from streaming interactions between them.

Ion and electron distributions change dramatically as we move toward the polar cap boundary. The ion conic heating is much more intense in regions of upgoing electron beams. The burst panels on the right show data from a region that includes electron solitary waves as well as energetic electron beams and energetic conics. The pitch angle plot at the bottom center is a $1.5 \mathrm{~s}$ average of ion data from the center of the most intense heating event at 21:05:30 UT. The peak energy of the ions extends to $20 \mathrm{keV}$, and the pitch angles lie generally between the ionospheric loss cone and $90^{\circ}$, with the peak energy at intermediate angles. Another conic pitch angle plot in the lower right box is a period of somewhet less intense heating. Both of these conic events display a rather high minimum energy which suggests that they are trapped by the potential that is accelerating the electron beams. The ions can only escape when they have gained sufficient 'perpendicular momentum' for their magnetic mirror force to overcome the parallel electric field.

\section{Summary}

The FAST electron and ion plasma analyzers were designed to measure auroral particle velocity distributions with an unprecendented combination of time, energy, and pitch angle resolution. Data are selected by on-board evaluation of the measurements to make the best use of the available telemetry rate. Measurements during these burst events can resolve ion and electron variations on ion cyclotron time scales and spatial scales of the electron inertial length. The on-orbit performance of the instrument has met all expectations. 


\section{Acknowledgements}

The authors are grateful to the many people who contributed to the success of this project. We give special recognition to Roland Scholz, who played a major role in assembling the FAST hardware, and was also instrumental in building many of the rocket and satellite experiments that led up to this mission.

\section{References}

Boehm, M., Paschmann, G., Clemmons, J., Hoefner, H., Frenzen, R., Ertl, M., Haerendel, G., Hill, P., Lauche, H., Eliasson, L. and Lundin, R.: 1994, 'The TESP Electron Spectrometer and Correlator (F7) on Freja', Space Sci. Rev. 70, 509.

Carlson, C. W., Curtis, D. W., Paschmann, G., and Michael, W.: 1983, in D. McDiarmid (ed.), 'An Instrument for Rapidly Measuring Plasma Distribution Functions with High Resolution', Adv. Space Res. 2, 67.

Carlson, C. W., Pfaff, R. W., and Watzin, J. G.: 1998a, ‘The Fast Auroral Snapshot (FAST) mission', Geophys. Res. Lett., 25, 2013.

Carlson, C. W. and McFadden, J. P.: 1998, in R. F. Pfaff, J. E. Borovsky, and D. T. Young (eds.), 'Design and Application of Imaging Plasma Instruments', Measurement Techniques in Space Plasmas: Particles, Geophys. Monogr. 102, AGU, Washington, p. 125.

Ergun, R. E., Carlson, C. W., Mozer, F. S., Delory, G. T., Temerin, M., McFadden, J. M., Pankow, D., Abiad, R., Harvey, P., Wilkes, R., Primbsch, H., Elphic, R., Strangeway, R., Pfaff, R., and Cattell, C. A.: 2000, 'The FAST Satellite Electric Field and Magnetic Field Instruments', Space Sci. Rev., this issue.

Gurnett, D. A.: 1974, 'The Earth as a Radio Source: Terrestrial Kilometric Radiation', J. Geophys. Res. 79, 4227.

Harvey, P. R., Curtis, D. W., Heetderks, H. D., Pankow, D., Rauch-Leiba, J. M., Wittenbrock, S. K., and McFadden, J. P.: 2001, 'The FAST Spacecraft Instrument Data Processing Unit', Space Sci. Rev., this issue.

Klumpar, D. M.: 1986, in T. Chang (ed.), 'A Digest and Comprehensive Bibliography on Transverse Auroral Ion Acceleration', Ion Acceleration in the Magnetosphere and Ionosphere, Geophys. Monogr. 38, AGU, Washington, p. 389.

McFadden, J. P., Carlson, C. W., and Boehm, M. H.: 1986, 'Field-aligned Electron Precipitation at the Edge of an Arc', J. Geophys. Res. 91, 1723.

McFadden, J. P., Carlson, C. W., Boehm, J. H., and Hallinan, T. J.: 1987, 'Field-aligned Electron Flux Oscillations that Produce Flickering Aurora', J. Geophys. Res. 92, 11133.

McFadden, J. P. and Carlson, C. W.: 1998, in R. F. Pfaff, J. E. Borovsky, and D. T. Young (eds.), 'Computer Simulation in Designing Electrostatic Optics for Space Plasma Experiments', Measurement Techniques in Space Plasmas: Particles, Geophys. Monogr. 102, AGU, Washington, p. 249.

McFadden, J. P., Carlson, C. W., Ergun, R. E., Chaston, C. C., Mozer, F. S., Temerin, M., Klumpar, D. M., Shelley, E. G., Peterson, W. K., Moebius, E., Kistler, L., Elphic, R., Strangeway, R., Cattell, C., and Pfaff, R.: 1998b, 'Electron modulation and ion cyclotron waves observed by FAST', Geophys. Res. Lett. 25, 2045.

McFadden, J. P., Carlson, C. W., and Ergun, R. E.: 1999, 'Microstructure of the auroral acceleration region as observed by FAST', J. Geophys. Res. 104, 14453.

McFadden, J. P., Ergun, R. E., Carlson, C. W., Herrick, W., Loran, J., Vernetti, J., Teitler, W., Bromund, K., and Quinn, T.: 2001, 'Science Operations and Data Handling for the FAST Satellite', Space Sci. Rev., this issue. 
Mozer F. S., Carlson, C. W., Hudson, M. K., Torbert, R. B., Parady, B. Yatteau, J., and Kelley, M. C.: 1977, 'Observation s of paired Electrostatic Shocks in the Polar Magnetosphere', Phys. Rev. Lett. 38, 292.

Pankow, D., Besuner, R., Ullrich, R., and Wilkes, R.: 2001, 'Deployment Mechanisms on the FAST Satellite: Radial Wires, Stiff Axials, and Magnetometer Booms', Space Sci. Rev., this issue.

Pfaff, R. , Carlson, C. W., Watzin, J., Everett, D., and Gruner, T.: 2001, 'An overview of the FAST Auroral Snapshot (FAST) Mission', Space Sci. Rev., this issue.

Sharp, R. D., Shelley, E. G., Johnson, R. G., and Ghielmetti, A. G.: 1980, 'Counterstreaming Electron Beams at Altitudes of $\sim 1$ RE over the Auroral Zone', J. Geophys. Res. 85, 92.

Strangeway, R. J., Kepko, L., Elphic, R. C., Carlson, C. W., Ergun, R. E., McFadden, J. P., Peria, W. J., Delory, G. T., Chaston, C. C., Temerin, M., Cattell, C. A., Moebius, E., Kistler, L. M., Klumpar, D. M., Peterson, W. K., Shelley, E. G., and Pfaff, R. F.: 1998, 'FAST observations of VLF waves in the auroral zone: Evidence of very low plasma densities', Geophys. Res. Lett. 25, 2065.

Temerin, M. A., Carlson, C. W., Cattell, C. A., Ergun, R. E., McFadden, J. P., Mozer, F. S., Klumpar, D. M., Peterson, W. K., Shelley, E. G., and Elphic, R. C.: 1990, in T. Chang (ed.), 'Waveparticle Interactions on the FAST Satellite', Physics of Space Plasmas, Scientific Publishers, Inc., Cambridge, MA., p. 343. 